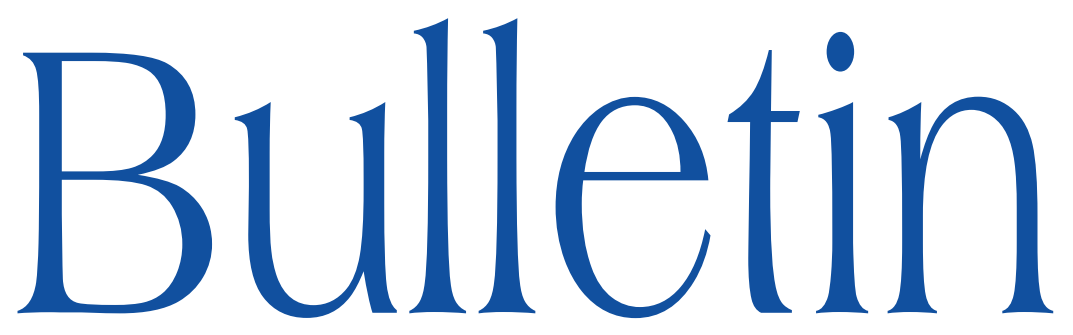

de la SOCIÉTÉ MATHÉMATIQUE DE FRANCE

\title{
LAGRANGIAN FIBRATIONS ON GENERALIZED KUMMER VARIETIES
}

\author{
Anne Duval \& Julien Roques
}

Tome 136 Fascicule 1

2008 


\title{
FAMILLES FUCHSIENNES D'ÉQUATIONS AUX (q-)DIFFÉRENCES ET CONFLUENCE
}

\author{
par Anne Duval \& Julien Roques
}

\begin{abstract}
Résumé. - On commence par présenter une méthode de résolution d'une famille de systèmes fuchsiens d'opérateurs de pseudo-dérivations associées à une famille à deux paramètres d'homographies, qui unifie et généralise les cas connus des systèmes différentiels, aux différences ou aux $q$-différences. Nous traitons ensuite dans cette famille des problèmes de confluence que l'on peut voir comme des problèmes de continuité en ces deux paramètres.
\end{abstract}

Abstract (Fuchsian families of equations). - In a first part, we give a method for solving a family of Fuchsian systems of operators of pseudo-derivations associated to a family of homographies with two parameters which unify and generalize the differential, the difference and the $q$-difference cases. In a second part, we study the problems of confluence related to these families.

Texte reçu le 4 août 2006, révisé le 7 mai 2007 et le 5 octobre 2007

Anne Duval, Université de Lille 1, UFR de Mathématiques Pures et Appliquées, Bâtiment M2, Cité Scientifique, 59655 Villeneuve d'Ascq Cedex - E-mail : Anne.Duval@univlille1.fr

Julien Roques, Laboratoire Émile Picard, Université Paul Sabatier, U.F.R. M.I.G., 118, route de Narbonne, 31062 Toulouse Cedex 4, France tlse.fr

Classification mathématique par sujets (2000). - 39A13, 41A58, 65Q05.

Mots clefs. - Équations fonctionnelles, fonctions spéciales, confluence, séries de $(q-)$ factorielles. 


\section{Introduction}

1.1. Problématique. - L'étude locale des trois types de systèmes linéaires

1) différentiel : $Y^{\prime}(x)=A(x) Y(x)$,

2) aux $q$-différences : $Y(q x)=A(x) Y(x)$,

où $q$ est un nombre complexe non nul de module différent de 1 ,

3) aux différences : $Y(x-1)=A(x) Y(x)$,

où $x$ est une variable complexe et $A(x)$ une matrice de fonctions, fait apparaître de grandes similitudes. Nous nous restreindrons dans ce qui suit à l'étude locale de ces systèmes au voisinage de l'infini qui est le seul point fixe, sur la sphère de Riemann, de la translation $x \mapsto x-1$ et l'un des deux points fixes de l'homographie $x \mapsto q x$.

Une matrice $T(x)$ inversible au voisinage de l'infini définit une «transformation de jauge », c'est-à-dire associe à la matrice $A(x)$ la matrice $B(x)$ du système que vérifie $X(x)=T(x) Y(x)$ lorsque $Y(x)$ vérifie le système de matrice $A(x)$. On a :

1) $B(x)=T(x)^{-1} A(x) T(x)-T(x)^{-1} T^{\prime}(x)$ dans le cas différentiel,

2) $B(x)=T(q x)^{-1} A(x) T(x)$ dans le cas des $q$-différences,

3) $B(x)=T(x-1)^{-1} A(x) T(x)$ dans le cas des différences.

Énonçons quelques résultats sous une forme faisant ressortir le parallélisme.

1) Supposons que $A(x)$ est la somme d'une série $\sum_{s \geq 0} A_{s} x^{-s-1}$, à coefficients dans l'anneau $M_{n}(\mathbb{C})$ des matrices constantes de dimension $n \times n$, convergente dans un voisinage de l'infini. Le système différentiel de matrice $A(x)$ est fuchsien. Il est en outre non résonnant si deux valeurs propres distinctes de la matrice $A_{0}$ ont une différence non entière. Dans ce cas, il existe une (unique) transformation de jauge tangente à l'identité donnée par une série

$$
T(x)=I+\sum_{s \geq 1} T_{s} x^{-s}
$$

convergente au voisinage de l'infini, telle que $B(x)=A_{0} x^{-1}$.

2) Supposons que $A(x)$ est la somme d'une série $\sum_{s \geq 0} A_{s} x^{-s}$, à coefficients dans l'anneau $M_{n}(\mathbb{C})$ des matrices constantes de dimension $n \times n$, convergente dans un voisinage de l'infini, avec $A_{0}$ inversible. Le système aux $q$-différences de matrice $A(x)$ est fuchsien. Il est non résonnant si deux valeurs propres distinctes de $A_{0}$ ne sont pas congrues modulo $q^{\mathbb{Z}}$ et dans ce cas une (unique) transformation de jauge tangente à l'identité le transforme en le système de matrice constante $A_{0}$, (voir [10, p. 1033] où le problème est traité au voisinage de 0 ; on s'y ramène par le changement de variable $x \leftarrow 1 / x)$. 
3) Afin d'obtenir des résultats analogues pour les sytèmes aux différences, il convient de remplacer les séries de puissances par les «séries de factorielles ». On suppose

$$
A(x)=I+\sum_{s \geq 0} A_{s} \frac{1}{x(x+1) \cdots(x+s)} .
$$

Le domaine naturel de convergence d'une telle série est un demi-plan vertical $\operatorname{Re} x \gg 0$ que l'on peut considérer comme un voisinage de $+\infty$. Comme dans le cas différentiel, le système est dit non résonnant si deux valeurs propres distinctes de $A_{0}$ ne sont pas congrues modulo $\mathbb{Z}$. Dans ce cas il existe une (unique) transformation de jauge tangente à l'identité donnée par une série de factorielles convergente $T(x)=I+\sum_{s \geq 0} T_{s} / x(x+1) \cdots(x+s)$ telle que $B(x)=I-A_{0} /(x-1)$. Remarquons que le système aux différences défini par $B(x)$ peut s'écrire

$$
(x-1)[Y(x)-Y(x-1)]=A_{0} Y(x) .
$$

Dans chaque cas, résoudre le sytème revient alors à résoudre un système à coefficients constants, ce qui se fait en réduisant cette matrice sous forme normale de Jordan puis en introduisant une famille de «caractères » et de « logarithmes » adaptés à chaque cas.

Par ailleurs ces trois familles de systèmes sont reliées entre elles par des propriétés de « confluence». En effet en notant $\sigma_{q}$ (resp. $\tau_{h}$ ) l'opérateur agissant sur une fonction $f$ par $\sigma_{q} f(x)=f(q x)\left(\operatorname{resp} . \tau_{h} f(x)=f(x+h)\right)$, on a

$$
\lim _{q \rightarrow 1} \frac{\sigma_{q}-\mathrm{id}}{(q-1) x}=\frac{\mathrm{d}}{\mathrm{d} x} \quad \text { et } \quad \lim _{h \rightarrow 0} \frac{\tau_{h}-\mathrm{id}}{h}=\frac{\mathrm{d}}{\mathrm{d} x} .
$$

L'étude de ces confluences, en liaison avec la théorie de Galois, a été menée récemment par J.Sauloy [10] pour la première famille et par le second auteur [8] pour la seconde. Ces deux auteurs montrent comment réaliser les deux étapes (transformation de jauge pour se ramener au cas constant et détermination d'un système fondamental de solutions dans le cas constant) d'une façon suffisamment canonique pour qu'un passage à la limite soit possible. Dans le premier cas le paramètre complexe $q$ est de module strictement plus grand que 1 et tend vers 1 le long d'une spirale logarithmique : $q=q_{0}^{\varepsilon}$ où $\left|q_{0}\right|>1$ et $\varepsilon$ est un réel positif que l'on fait tendre vers 0 . Dans le second cas $h$ est réel positif et tend vers 0 .

Un autre type de confluence a été considéré par le premier auteur dans [3] : on part de la famille d'opérateurs définis par $\sigma_{q, 1} f(x)=f(q x+1)$ et on étudie la convergence $\lim _{q \rightarrow 1} \sigma_{q, 1}=\tau_{1}$ notée $\tau$. Il s'agit cette fois d'un phénomène de confluence d'opérateurs associés à une famille d'homographies à deux points fixes confluant vers une homographie à un point fixe. Rappelons le fait bien connu que tout système $Y(\psi(x))=A(x) Y(x)$ où $\psi$ est une homographie de 
la sphère de Riemann distincte de l'identité, se ramène par changement de variable homographique soit à un système aux $q$-différences (si $\psi$ a deux points fixes) soit à un système aux différences (si $\psi$ a un point fixe). Ainsi le système $Y(q x+1)=A_{q}(x) Y(x)$ devient un système aux $q$-différences si on effectue le changement de variable $x \mapsto(q-1) x+1$. Au niveau de l'opérateur, la confluence précédente est seulement une spécialisation en $q=1$, mais cette spécialisation n'est pas possible dans les solutions obtenues par le changement de variable indiqué. Dans l'article cité l'étude de cette confluence est faite en remarquant qu'il est possible d'exprimer toute série convergente à l'infini en une série de la forme $a_{0}+\sum_{s \geq 1} a_{s} /(x ; q)_{s}$ où $(x ; q)_{s}=\prod_{j=0}^{s-1}\left(1-q^{j} x\right)$ (voir [2, p. 344]). En supposant que $q$ est réel et tend vers 1 par valeurs supérieures, on montre dans [3] qu'après une telle écriture suivie du changement de variable indiqué, les solutions obtenues tendent vers celles du système aux différences limite.

Dans le présent travail, nous généralisons et unifions ces trois articles en considérant une famille de systèmes associés à la famille d'opérateurs $\sigma_{q, h} f(x)=f(q x+h)$. Nous montrons qu'il est possible, sous certaines hypothèses, de définir une solution canonique pour le système

$$
Y(q x+h)=A^{(q, h)}(x) Y(x) .
$$

Celle-ci est obtenue en résolvant chaque système de la famille en les deux étapes indiquées : par une transformation de jauge tangente à l'identité définie par une série appropriée (séries de $(q, h)$-factorielles) convergente dans un domaine convenable, on se ramène au cas d'un système à coefficients constants que l'on résout à l'aide d'une famille adaptée de fonctions. De plus ces deux étapes sont menées en contrôlant la dépendance en $(q, h)$. Cette étude aboutit au résultat énoncé dans le théorème 1 , section 1.3. On étudie ensuite les trois types de confluence $:(q, h) \rightarrow\left(q_{0}, 0\right)\left(q_{0}>1\right),(q, h) \rightarrow\left(1, h_{0}\right)\left(h_{0}>0\right)$ et $(q, h) \rightarrow(1,0)$. Les conclusions obtenues sont l'objet du théorème 2 , section 1.3.

Indiquons le plan de l'article. Après avoir donné les définitions et notations utiles, nous énonçons les deux théorèmes principaux de notre article. Un premier paragraphe étudie les séries de $(q, h)$-factorielles. Chacun des deux paragraphes suivants est consacré à la preuve de l'un des théorèmes.

1.2. Notations. - Dans tout ce travail $q$ est un réel supérieur ou égal à 1 dont l'inverse est noté $p$ et $h$ est un réel positif ou nul.

Rappelons, pour $q \neq 1$ et $\lambda \in \mathbb{C}$, la notation de Jackson

$$
[\lambda]_{q}=\frac{q^{\lambda}-1}{q-1},
$$

que l'on étend au cas $q=1$ par $[\lambda]_{1}=\lambda$. On pose

$$
{ }_{h}[\lambda]_{q}=h[\lambda]_{q}
$$

TOME $136-2008-\mathrm{N}^{\mathrm{O}} 1$ 
On note

$$
\mathcal{Q}=] 1,+\infty[\times] 0,+\infty\left[, \quad \mathcal{Q}^{+}=\left[1,+\infty\left[\times\left[0,+\infty\left[, \quad \mathcal{Q}^{*}=\mathcal{Q}^{+} \backslash\{(1,0)\} .\right.\right.\right.\right.\right.
$$

Pour $(q, h) \in \mathcal{Q}^{+}$et $s \in \mathbb{N}^{*}$, nous introduisons la fraction rationnelle

$$
x^{-{ }_{h}[s]} q=\frac{1}{x\left(q x+{ }_{h}[1]_{q}\right) \cdots\left(q^{s-1} x+{ }_{h}[s-1]_{q}\right)} .
$$

On écrit aussi $x^{-h^{[0]}} q=1$ et on abrège $x^{-{ }_{1}[s]} q$ en $x^{-[s]_{q}}$ et $x^{-{ }_{1}[s]} 1$ en $x^{-[s]}$. Remarquons que $x^{-{ }_{0}[s]} q=q^{-\frac{1}{2} s(s-1)} x^{-s}$.

Afin de faire ressortir les analogies mentionnées dans l'introduction, si $(q, h) \neq(1,0)$, il sera utile de remplacer l'opérateur $\sigma_{q, h}$ par la pseudodérivation

$$
\Delta_{q, h}=\frac{\sigma_{q, h}^{-1}-\mathrm{id}}{\sigma_{q, h}^{-1}(x)-x}=\frac{\sigma_{p,-p h}-\mathrm{id}}{(p-1) x-p h} .
$$

Cet opérateur vérifie la «formule de Leibniz »

$$
\begin{aligned}
\Delta_{q, h}(f g)(x) & =\sigma_{p,-p h} f(x) \Delta_{q, h} g(x)+g(x) \Delta_{q, h} f(x) \\
& =\sigma_{p,-p h} g(x) \Delta_{q, h} f(x)+f(x) \Delta_{q, h} g(x) .
\end{aligned}
$$

L'analogue de l'opérateur « fuchsien » $x \mathrm{~d} / \mathrm{d} x$ est alors l'opérateur

$$
{ }_{p} \delta_{h}=p(x-h) \Delta_{q, h} .
$$

On convient naturellement que ${ }_{1} \delta_{0}=x \mathrm{~d} / \mathrm{d} x$.

Le résultat suivant est une généralisation immédiate de la proposition 1.1 de [2] .

LEMME 1. - La fraction rationnelle $x^{-{ }^{[s]}}$ est un vecteur propre de ${ }_{p} \delta_{h}$ pour la valeur propre $-[s]_{q}$.

Nous envisageons dans ce travail des familles de systèmes aux $(q, h)$-différences

$$
{ }_{p} \delta_{h} Y(x)=A^{(q, h)}(x) Y(x), \quad(q, h) \in \mathcal{Q}^{+}
$$

où $A^{(q, h)}(x)$ est une série de la forme

$$
A^{(q, h)}(x)=\sum_{s=0}^{+\infty} A_{s}^{(q, h)} x^{-{ }_{h^{[s]}}} q
$$

avec $A_{s}^{(q, h)} \in M_{n}(\mathbb{C})$. De telles séries sont appelées séries de $(q, h)$-factorielles formelles à coefficients dans $M_{n}(\mathbb{C})$.

Des conditions suffisantes de convergence de ces séries sont données au paragraphe 2 et rendront naturelles les définitions qui suivent. 
Pour $\lambda, x \in \mathbb{C}$, la signification habituelle, lorsque $(q, h) \in \mathcal{Q}$, de l'expression

$$
\left|x-\frac{h}{1-q}\right|>\left|\lambda-\frac{h}{1-q}\right|
$$

est prolongée à $\mathcal{Q}^{+}$en convenant qu'elle signifie $\operatorname{Re}(x)>\operatorname{Re}(\lambda)$ si $q=1, h>0$ et $|x|>|\lambda|$ si $h=0$. Pour $\lambda \in \mathbb{C}$, on note

$$
V^{(q, h)}(\lambda)=\left\{x \in \mathbb{C} ;\left|x-\frac{h}{1-q}\right|>\left|\lambda-\frac{h}{1-q}\right|\right\} .
$$

Notons que la famille $\left(V^{(q, h)}(\lambda)\right)_{\lambda \in \mathbb{R}^{+}}$constitue un système fondamental de voisinages de $\infty$ sauf si $h \neq 0$ et $q=1$ où il s'agit de voisinages de $+\infty$.

On suppose choisies une norme notée $\|\cdot\|$ sur $M_{n}(\mathbb{C})$ et une norme $|\cdot|$ sur $\mathbb{C}^{n}$ telles que si $A \in M_{n}(\mathbb{C})$ et $X \in \mathbb{C}^{n}$, on a $|A X| \leq\|A\| \cdot|X|$. On supposera aussi par commodité que $\|I\|=1$, si $I$ désigne la matrice identité.

La définition suivante fixe le cadre technique dans lequel nous nous placerons.

DÉfinition 1. - Soient $C$ et $\lambda$ deux réels positifs et $\Lambda \subset \mathcal{Q}^{+}$. Une famille de matrices $\left(A^{(q, h)}(x)\right)_{(q, h) \in \Lambda}$ est dite fuchsienne $(C, \lambda)$ sur $\Lambda$ si, pour tout $(q, h) \in \Lambda$, la matrice $A^{(q, h)}(x)$ est une série de $(q, h)$-factorielles à coefficients dans $M_{n}(\mathbb{C})$

$$
A^{(q, h)}(x)=\sum_{s \geq 0} A_{s}^{(q, h)} x^{-{ }_{h}[s]} q
$$

et si, pour tout $s \geq 1$,

$$
\left\|A_{s}^{(q, h)}\right\| \leq C q^{s-1}\left(\lambda^{\left.-h^{[s-1}\right]_{q}}\right)^{-1} .
$$

Une famille de systèmes (1) est dite fuchsienne $(C, \lambda)$ sur $\Lambda$ si

1) pour tout $(q, h) \in \Lambda$, la matrice $I+(1-q) A_{0}^{(q, h)}$ est inversible,

2) la famille de matrices $\left(A^{(q, h)}(x)\right)_{(q, h) \in \Lambda}$ est fuchsienne $(C, \lambda)$ sur $\Lambda$.

Elle est dite non résonnante si, pour toute valeur propre $c$ de $A_{0}^{(q, h)}, q^{s} c-[s]_{q}$ n'est valeur propre de $A_{0}^{(q, h)}$ pour aucun entier $s \neq 0$.

En particulier, chaque système d'une telle famille fuchsienne est fuchsien dans les sens classiques : lorsque $h=0$, la définition cö̈ncide avec celle de système fuchsien en $\infty$ au sens habituel et lorsque $q=h=1$, c'est la définition d'un système de première espèce en $+\infty$ de [6].

Notons que la condition d'inversibilité de la matrice $I+(1-q) A_{0}^{(q, h)}$ assure que la matrice du système (1) écrit sous la forme $Y(q x+h)=B(x) Y(x)$ est inversible, en accord avec le fait que $\sigma_{q, h}$ est un automorphisme. Elle est évidemment réalisée si $q=1$.

Remarquons que la condition de non-résonance ne fait pas intervenir la valeur de $h$. Lorsque $q=1$, c'est la condition classique : deux valeurs propres 
distinctes ne différent pas d'un entier non nul. Lorsque $q>1$, en remarquant que $q^{s}[\tilde{c}]_{q}+[s]_{q}=[s+\tilde{c}]_{q}$ pour $\tilde{c} \in \mathbb{C}$, on voit qu'en notant les valeurs propres de $A_{0}^{(q, h)}$ sous la forme $c=-[-\tilde{c}]_{q}$, ce qui est licite vue la condition d'inversibilité indiquée, la non-résonance équivaut à ce que $\tilde{c}$ et $\tilde{d}$ ne sont pas congrus modulo $\mathbb{Z}$ lorsque $\tilde{c} \neq \tilde{d}$. On retrouve bien la condition de non-résonance rappelée plus haut.

Nous terminons cette section en introduisant la famille de fonctions que nous utiliserons pour résoudre les systèmes à matrice constante.

Pour tout $x \in \mathbb{C}$, si $s \in \mathbb{N}^{*}$, on pose

$$
(x, p)_{s}=\prod_{j=0}^{s-1}\left(1-p^{j} x\right) \quad \text { et } \quad(x ; p)_{\infty}=\lim _{s \rightarrow \infty}(x ; p)_{s} .
$$

Nous utiliserons aussi la fonction thêta de Jacobi suivante dont les propriétés de base sont rappelées en 3.1.1:

$$
\Theta_{q}(x)=\sum_{n \in \mathbb{Z}}(-1)^{n} q^{-\frac{1}{2} n(n-1)} x^{n} .
$$

Pour $\alpha \in \mathbb{C}^{*}$, on note $\Theta_{q, \alpha}(x)=\Theta_{q}\left(\alpha^{-1} x\right)$.

Nous noterons $\Gamma$ la fonction Gamma d'Euler.

Définition 2. - Pour $(q, h) \in \mathcal{Q}^{+}$et $c \in \mathbb{C}$, on note $e_{c}^{(q, h)}(x)$ la fonction

$$
e_{c}^{(q, h)}(x)= \begin{cases}\left(\frac{h}{1-p}\right)^{c} \frac{((1-p) x / h+p ; p)_{\infty}}{\left(p^{c}((1-p) x / h+p) ; p\right)_{\infty}} & \text { si } q>1, h>0, \\ h^{c} \Gamma(1+c-x / h) / \Gamma(1-x / h) & \text { si } q=1, h>0, \\ \Theta_{q}(x) / \Theta_{q, q^{c}}(x) & \text { si } q>1, h=0, \\ (-x)^{c} & \text { si }(q, h)=(1,0) .\end{cases}
$$

Dans le dernier cas, on suppose fixée une détermination du logarithme et on abrège $e_{c}^{(1,0)}$ en $e_{c}$.

Pour tout entier $k \geq 0$, on pose

$$
\ell_{c, k}^{(q, h)}(x)=\frac{1}{k !} \frac{\partial^{k}}{\partial c^{k}} e_{c}^{(q, h)}(x) .
$$

Soient $r \geq 1$ un entier et $E_{i, j}$ la matrice carrée élémentaire de dimension $r$ dont tous les éléments sont nuls sauf celui situé sur la ligne $i$ et la colonne $j$ qui vaut 1 . On note

$$
N_{r}=\sum_{i=1}^{r-1} E_{i, i+1}
$$


Pour $c \in \mathbb{C}$ et $(q, h) \in \mathcal{Q}^{+}$, on définit les matrices $r \times r$

$$
\mathcal{L}_{c, r}^{(q, h)}(x)=\sum_{j=0}^{r-1} \ell_{c, j}^{(q, h)}(x) N_{r}^{j} \quad \text { et } \quad A_{c, r}^{(q, h)}=-[-c]_{q} I_{r}+\frac{q^{-c}}{1-q}\left(q^{-N_{r}}-I_{r}\right)
$$

Soient $r_{1}, \ldots, r_{m}$ des entiers strictement positifs de somme $n$ et $c_{1}, \ldots, c_{m}$ des nombres complexes; on note

$$
J_{0}^{(q, h)}=\operatorname{diag}\left(A_{c_{1}, r_{1}}^{(q, h)}, \ldots, A_{c_{m}, r_{m}}^{(q, h)}\right) .
$$

Soit $P$ une matrice constante inversible et $A_{0}^{(q, h)}=P J_{0}^{(q, h)} P^{-1}$.

DÉfinition 3. - Avec les notations précédentes, on définit les matrices $n \times n$ $\mathcal{E}_{J_{0}^{(q, h)}}(x)=\operatorname{diag}\left(\mathcal{L}_{c_{1}, r_{1}}^{(q, h)}(x), \ldots, \mathcal{L}_{c_{m}, r_{m}}^{(q, h)}(x)\right) \quad$ et $\quad \mathcal{E}_{A_{0}^{(q, h)}}(x)=P \mathcal{E}_{J_{0}^{(q, h)}}(x) P^{-1}$.

1.3. Les deux théorèmes. - Une transformation de jauge de matrice $F(x)$ transforme le système ${ }_{p} \delta_{h} Y(x)=A(x) Y(x)$ en le système de matrice

$$
A^{F}(x)=F(p x-p h)^{-1}\left[A(x) F(x)-{ }_{p} \delta_{h} F(x)\right] .
$$

Il s'agit de la matrice codant le système obtenu à partir de ${ }_{p} \delta_{h} Y(x)=A(x) Y(x)$ par le changement de fonction inconnue $X(x)=F(x) Y(x)$. Nous utiliserons des transformations de jauges développables en séries de $(q, h)$-factorielles tangentes à l'identité, c'est-à dire de la forme

$$
F^{(q, h)}(x)=I+\sum_{s \geq 1} F_{s}^{(q, h)} x^{-{ }^{[s]}} q .
$$

Énonçons le premier de nos deux résultats principaux.

ThÉORÈme 1. - Soit $\Lambda$ une partie bornée de $\mathcal{Q}^{+}$. Soit une famille fuchsienne non résonnante $(C, \lambda)$ sur $\Lambda$ de systèmes (1) où

$$
A^{(q, h)}(x)=\sum_{s \geq 0} A_{s}^{(q, h)} x^{-{ }^{[s]} q} .
$$

On suppose que, pour tout $(q, h) \in \Lambda$, il existe $P^{(q, h)} \in \mathrm{Gl}_{n}(\mathbb{C})$ telle que

$$
P^{(q, h)} J_{0}^{(q, h)}=A_{0}^{(q, h)} P^{(q, h)}
$$

où

$$
J_{0}^{(q, h)}=\operatorname{diag}\left(A_{c_{1}, r_{1}}^{(q, h)}, \ldots, A_{c_{m}, r_{m}}^{(q, h)}\right)
$$

avec $c_{1}, \ldots, c_{m}$ des nombres complexes vérifiant $c_{i}-c_{j} \notin \mathbb{Z}^{*}$ et $r_{1}, \ldots, r_{m}$ des entiers strictement positifs tels que $r_{1}+\cdots+r_{m}=n$. Si les familles $\left(P^{(q, h)}\right)_{(q, h) \in \Lambda}$ et $\left(\left(P^{(q, h)}\right)^{-1}\right)_{(q, h) \in \Lambda}$ sont bornées, alors : 
1) Pour tout $(q, h) \in \Lambda$, il existe une unique transformation de jauge formelle tangente à l'identité $F^{(q, h)}(x)$, définie par une série de $(q, h)$ factorielles de terme constant $I$, telle que

$$
\left(A^{(q, h)}\right)^{F^{(q, h)}}(x)=A_{0}^{(q, h)} .
$$

2) Il existe $\left(C^{\prime}, \lambda^{\prime}\right)$ tels que la famille $\left(F^{(q, h)}(x)\right)_{(q, h) \in \Lambda}$ est fuchsienne $\left(C^{\prime}, \lambda^{\prime}\right)$.

3) Les deux propriétés suivantes sont satisfaites :

(a) pour toute matrice $P \in \mathrm{Gl}_{n}(\mathbb{C}), F^{(q, h)}(x) P \mathcal{E}_{J_{0}^{(q, h)}}(x)$ est une matrice fondamentale de solutions dans $V^{(q, h)}\left(\lambda^{\prime}\right)$ du système (1) si et seulement si $P J_{0}^{(q, h)}=A_{0}^{(q, h)} P$,

(b) la matrice $\mathcal{X}_{\mathrm{can}}^{(q, h)}(x)=F^{(q, h)}(x) \mathcal{E}_{A_{0}^{(q, h)}}(x)$ est, dans $V^{(q, h)}\left(\lambda^{\prime}\right)$, une matrice fondamentale de solutions de (1), indépendante du choix de la matrice $P$ conjuguant $A_{0}^{(q, h)}$ à sa forme normale.

DÉFInition 4. - La famille de matrices $\left(\mathcal{X}_{\mathrm{can}}^{(q, h)}(x)\right)_{(q, h) \in \Lambda}$ définie dans le théorème précédent est appelée solution canonique de la famille (1).

Avant d'énoncer des propriétés de confluence pour les fonctions, décrivons le comportement de leurs domaines de définition. Pour $\lambda>0$ fixé, la famille $V^{(q, h)}(\lambda)$ vérifie les propriétés suivantes de continuité en un point du bord de $\mathcal{Q}^{+}$:

1) Pour $q_{0}>1$ fixé, la famille $V^{\left(q_{0}, h\right)}(\lambda)$ croît lorsque $h \downarrow 0$ et

$$
\bigcup_{h>0} V^{\left(q_{0}, h\right)}(\lambda)=\{|x|>\lambda\}=V^{\left(q_{0}, 0\right)}(\lambda) .
$$

Notons que cette limite est indépendante de $q_{0}>1$ et que le résultat est valable si $(q, h) \rightarrow\left(q_{0}, 0\right)$ avec $q_{0}>1$.

2) Pour $h_{0}>0$ fixé, la famille $V^{\left(q, h_{0}\right)}(\lambda)$ décroît lorsque $q \downarrow 1$ et

$$
\bigcap_{q>1} V^{\left(q, h_{0}\right)}(\lambda)=\{\operatorname{Re} x>\lambda\}=V^{\left(1, h_{0}\right)}(\lambda) .
$$

Cette fois encore, le résultat persiste si $(q, h) \rightarrow\left(1, h_{0}\right)$ avec $h_{0}>0$.

3) La famille $V^{(q, h)}(\lambda)$ décroît avec $h /(q-1)$. Chaque $V^{(q, h)}(\lambda)$ contient le demi-plan $\operatorname{Re} x>\lambda$. Si $\Lambda^{\prime} \subset \Lambda$, l'intersection des $V^{(q, h)}(\lambda)$ pour $(q, h) \in \Lambda^{\prime}$ est un voisinage de $\infty$ si et seulement si la famille $(h /(q-1))_{(q, h) \in \Lambda^{\prime}}$ est bornée. Lorsqu'on fait l'hypothèse

$$
(q, h) \rightarrow(1,0), \quad \text { de sorte que } h /(q-1) \rightarrow \ell
$$

où $\ell \in[0,+\infty]$, alors $V^{(q, h)}(\lambda)$ a pour limite $\{|x+\ell|>\lambda+\ell\}$ si $\ell$ est fini et $\{\operatorname{Re} x>\lambda\}$ si $\ell=+\infty$. On note $V_{\ell}(\lambda)$ ce domaine limite. 
Nous étudierons la confluence des solutions (i.e. la continuité en les paramètres $(q, h))$ dans les trois cas suivants :

$\mathrm{C} 1:(q, h)$ tend vers $\left(q_{0}, 0\right)$ avec $q_{0}>1$,

$\mathrm{C} 2:(q, h)$ tend vers $\left(1, h_{0}\right)$ avec $h_{0}>0$,

C3 : $(q, h)$ tend vers $(1,0)$ et l'hypothèse $(\mathrm{H})$ est satisfaite.

Énonçons le deuxième résultat principal de cet article.

THÉORÈme 2. - Soit une famille (1) vérifiant les hypothèses du théorème 1 dont on reprend les notations. Soit $\left(q_{0}, h_{0}\right) \in \bar{\Lambda} \cap \partial \mathcal{Q}^{+}$. On suppose que :

1) pour tout $s \geq 0, A_{s}^{(q, h)}$ a une limite $A_{s}$ lorsque $(q, h) \in \Lambda$ tend vers $\left(q_{0}, h_{0}\right)$ de telle sorte que l'on soit dans l'une des situations $\mathrm{C} 1, \mathrm{C} 2, \mathrm{C} 3$ et, dans ce dernier cas, on suppose $\ell \in \mathbb{R}^{+*}$;

2) il existe des matrices de conjugaison $P^{(q, h)}$ telles que, quand $(q, h)$ tend vers $\left(q_{0}, h_{0}\right), P^{(q, h)}$ converge vers une matrice conjuguant $A_{0}^{\left(q_{0}, h_{0}\right)}$ $\grave{a} J_{0}^{\left(q_{0}, h_{0}\right)}$.

Alors :

1) la série de $\left(q_{0}, h_{0}\right)$-factorielles

$$
A(x)=\sum_{s \geq 0} A_{s} x^{-h_{0}[s]} q_{0}
$$

converge dans $V^{\left(q_{0}, h_{0}\right)}(\lambda)$ dans les cas $\mathrm{C} 1$ et $\mathrm{C} 2$, dans $V_{\ell}(\lambda)$ dans le cas $\mathrm{C} 3$;

2) si le système aux $\left(q_{0}, h_{0}\right)$-différences défini par $A(x)$ est fuchsien et non résonnant, alors

$\mathrm{C} 1$ : en posant $h_{n}=\left(1-p_{0}\right) p_{0}^{n}$, la suite $\left(\mathcal{X}_{\mathrm{can}}^{\left(q_{0}, h_{n}\right)}(x)\right)_{n \geq 0}$ converge vers $\mathcal{X}_{\text {can }}^{\left(q_{0}, 0\right)}(x)$, uniformément sur tout compact de $\left\{|x|>\lambda^{\prime}\right\} \backslash\left(c+h_{0} \mathbb{N}^{*}\right)$;

$\mathrm{C} 2$ : la famille $\mathcal{X}_{\mathrm{can}}^{(q, h)}(x)$ tend vers $\mathcal{X}_{\mathrm{can}}^{\left(1, h_{0}\right)}(x)$, uniformément sur tout compact de $\left\{\operatorname{Re} x>\lambda^{\prime}\right\} \backslash\left(c+h_{0} \mathbb{N}^{*}\right)$;

C3 : la famille $\mathcal{X}_{\mathrm{can}}^{(q, h)}(x)$ tend vers $\mathcal{X}_{\mathrm{can}}^{(1,0)}(x)$, uniformément sur tout compact de $V_{\ell}\left(\lambda^{\prime}\right) \backslash \mathbb{R}^{+}$.

Notons que dans le cas C1, la famille des caractères n'admet pas de limite lorsque $h$ tend vers 0 . En revanche, le théorème 4 , section 4.1 assure que cette famille est normale et donne l'ensemble de ses valeurs d'adhérence.

\section{Quelques propriétés des séries de $(q, h)$-factorielles}

Nous donnons ici quelques propriétés des séries de $(q, h)$-factorielles, i.e. des séries de la forme $\sum_{s \geq 0} A_{s} x^{-h^{[s]}} q$. 
2.1. Conditions suffisantes de convergence. - On a déjà remarqué que $x^{-{ }_{0}[s]} q=$ $q^{-\frac{1}{2} s(s-1)} x^{-s}$. Les séries de $(q, 0)$-factorielles sont donc les séries de puissances en $x^{-1}$. Lorsqu'il n'est pas vide, leur domaine de convergence absolue est de la forme $V^{(q, 0)}(\lambda)$ pour un réel positif $\lambda$.

Les séries de $(1,1)$-factorielles sont les séries de factorielles. Leur domaine de convergence absolue, s'il est non vide, est de la forme $V^{(1,1)}(\lambda)$ pour un réel positif $\lambda$ (voir [6] par exemple).

Enfin, les séries de $(q, 1)$-factorielles sont étudiées dans [2, p. 351] où elles sont appelées « séries de factorielles mixtes ». Toujours sous réserve de converger en au moins un point, elles convergent absolument dans un domaine $V^{(q, 1)}(\lambda)$ pour un réel positif $\lambda$.

Pour tout entier $s \geq 1$ et tout $h>0$ :

$$
x^{-h^{[s]}} q=h^{-s}\left(\frac{x}{h}\right)^{-[s]_{q}} .
$$

Lorsque $h \neq 0$, le changement de variable $x \mapsto x / h$ transforme donc une série de $(q, h)$-factorielles en une série de $(q, 1)$-factorielles. On en déduit qu'une série de $(q, h)$-factorielles qui converge en un point, converge absolument dans un domaine $V^{(q, h)}(\lambda)$ pour un réel positif $\lambda$.

Par ailleurs, il résulte de [6] pour les séries de factorielles et de [2, prop. 2.8] pour les séries de $(q, 1)$-factorielles que l'on peut énoncer le résultat général suivant.

Proposition 1. - Soit $\left(A^{(q, h)}(x)\right)_{(q, h) \in \Lambda}$ une famille fuchsienne $(C, \lambda)$ sur $\Lambda \subset \mathcal{Q}^{+}$. Pour tout $(q, h) \in \Lambda$ la série de $(q, h)$-factorielles $A^{(q, h)}(x)$ converge absolument dans $V^{(q, h)}(\lambda)$ et sa somme est une fonction holomorphe.

Une fonction $a$ à valeurs complexes, définie et holomorphe sur $V^{(q, h)}(\lambda)$, est dite développable en série de $(q, h)$-factorielles s'il existe une suite de nombres complexes $\left(a_{s}\right)_{s \geq 0}$ et un réel $\lambda^{\prime}>0$ tels que, pour tout $x \in V^{(q, h)}\left(\lambda^{\prime}\right)$,

$$
a(x)=\sum_{s=0}^{+\infty} a_{s} x^{-h^{[s]}} q .
$$

Lorsqu'il existe, un tel développement est unique. L'ensemble des fonctions développables en séries de $(q, h)$-factorielles est noté $\mathcal{O}_{f}^{(q, h)}$.

Notons qu'il s'agit d'un anneau commutatif unitaire et intègre pour l'addition et la multiplication usuelles des fonctions. De plus, l'application qui à $a \in \mathcal{O}_{f}^{(q, h)}$ associe $\widehat{a}(x) \in \mathbb{C}\left[\left[x^{-1}\right]\right]$, le développement de $a$ en l'infini considéré comme une série formelle en la variable $x^{-1}$, est un monomorphisme d'anneaux mais pas un épimorphisme. 
L'exemple de base est la fonction $1 /(x-\lambda)(\lambda \in \mathbb{C})$ qui admet le développement en série de $(q, h)$-factorielles convergente dans $V^{(q, h)}(\lambda)$ :

$$
\frac{1}{x-\lambda}=\sum_{s=1}^{\infty} q^{s-1}\left(\lambda^{-{ }_{h}[s-1]} q\right)^{-1} x^{-{ }_{h}[s]} q .
$$

Remarquons que si $\lambda$ est réel positif cette série est à coefficients réels positifs, ce qui permet de l'utiliser comme série majorante.

2.2. Formule de translation. - La combinatoire des séries de factorielles (formelles) est rendue possible par l'existence d'une « formule de translation » permettant d'exprimer une série en $x^{-[s]}$ comme série en $(x+\rho)^{-[s]}$. Un résultat analogue (formule d'homothétie) existe pour les séries de $(q, 1)$-factorielles (voir [2, p. 352]) et le changement de variable $x \mapsto x / h$ permet de l'obtenir pour les séries de $(q, h)$-factorielles avec $h>0$. On prouve en effet que, si $A(x)=A_{0}+\sum_{s \geq 1} A_{s} x^{-{ }_{h}[s]} q$, alors

$$
A(p x-p h)=A_{0}+\sum_{s \geq 1} q^{s}\left(A_{s}+[s-1] ! \sum_{k=1}^{s-1} h^{s-k} \frac{A_{k}}{[k-1] !}\right) x^{-{ }_{h}[s]} q
$$

où $[0] !=1$ et $[j] !=j !$ si $q=1,[j] !=\prod_{k=1}^{j}[k]_{q}$ si $j \geq 1$ (notation de Jackson). Si $h=0$, cette formule se vérifie sans recourir à une formule de translation.

2.3. Produit. - Lorsque $h \neq 0$, la formule de multiplication donnée ci-dessous permet d'exprimer le produit de deux séries de $(q, h)$-factorielles formelles comme une série de même nature. Ces formules figurent dans [6] dans le cas $(1,1)$, dans [2, prop. 2.9] dans le cas $(q, 1)$ et la formule ci-dessous en résulte.

Soient $A(x)=\sum_{s=0}^{+\infty} A_{s} x^{-{ }^{[s]}} q$ et $B(x)=\sum_{s=0}^{+\infty} B_{s} x^{-{ }{ }^{[s]}} q$ deux séries formelles de $(q, h)$-factorielles. Leur produit est la série formelle

$$
(A B)(x)=\sum_{s=0}^{+\infty} C_{s} x^{-{ }_{h}[s]} q
$$

avec $C_{0}=A_{0} B_{0}$ et, pour $s \geq 1$,

$$
C_{s}=A_{0} B_{s}+A_{s} B_{0}+\sum_{(i, j, k) \in J_{s}} h^{k} q^{k+i j} c_{i, j, k} A_{i} B_{j},
$$

où $J_{s}=\{(i, j, k) ; i, j \geq 1, k \geq 0, i+j+k=s\}$ et

$$
c_{i, j, k}=\frac{[i+k-1] ![j+k-1] !}{[i-1] ![j-1] ![k] !} .
$$

Notons que $c_{i, j, 0}=1$ pour tout $i, j$ et que cette formule se simplifie en l'habituelle lorsque $h=0$. 
Lorsque les séries $A(x)$ et $B(x)$ convergent, leur produit converge au moins dans le plus petit de leurs domaines de convergence.

2.4. Inverse. - Pour les séries de $(q, h)$-factorielles à coefficients dans $\mathbb{C}$, nous ferons usage du résultat suivant (voir [2]).

Proposition 2. - Soient $\lambda, \mu$ des réels positifs et $a(x)=1-\sum_{s=1}^{+\infty} a_{s} x^{-{ }^{[}{ }^{[s]}} q$ une série de $(q, h)$-factorielles dont les coefficients sont des nombres complexes vérifiant

$$
\left|a_{s}\right| \leq \mu q^{s-1}\left(\lambda^{-{ }_{h}[s-1]} q\right)^{-1} .
$$

Alors $b=1 / a$ est une série de $(q, h)$-factorielles de la forme

$$
b(x)=1+\sum_{s=1}^{+\infty} b_{s} x^{-{ }_{h}[s]} q \quad \text { avec } \quad\left|b_{s}\right| \leq \mu q^{s-1}\left((\lambda+\mu)^{-{ }_{h}[s-1]} q\right)^{-1} .
$$

\section{Preuve du théorème 1}

Comme indiqué plus haut, pour résoudre un système aux $(q, h)$-différences fuchsien, on se ramène au cas constant par une transformation de jauge convenable. On étudie d'abord un système de matrice constante (en $x$ ) non résonnante, puis on montre l'existence d'une unique transformation de jauge ramenant à ce cas. En vue de l'étude ultérieure de la confluence, cette deuxième étape est réalisée en famille (famille fuchsienne).

3.1. Cas d'une matrice constante. - Résoudre un système à matrice constante, revient à résoudre les deux familles d'équations suivantes :

— « équation des caractères » :

$$
{ }_{p} \delta_{h} y(x)=-[-c]_{q} y(x),
$$

— «équation des logarithmes » :

$$
{ }_{p} \delta_{h} \ell_{c, k}^{(q, h)}(x)=-[-c]_{q} \ell_{c, k}^{(q, h)}(x)+\sum_{j=1}^{k} \frac{(-1)^{j}}{j !} \frac{\ln ^{j} q}{1-q} q^{-c} \ell_{c, k-j}^{(q, h)}(x) .
$$


3.1.1. Caractères et logarithme. - Les fonctions $e_{c}^{(q, h)}(x)$ définies dans l'introduction sont méromorphes sur $\mathbb{C}$ lorsque $(q, h) \neq(1,0)$. Rappelons quelques propriétés fondamentales des fonctions spéciales utilisées pour les construire (à savoir : la fonction $\Gamma$, le symbole de Pochamer et la fonction $\Theta_{q}$ ). La fonction $\Gamma$ est méromorphe sur $\mathbb{C}$, sans zéros, à pôles simples aux entiers négatifs et vérifie $\Gamma(x+1)=x \Gamma(x)$. La fonction $(x ; p)_{\infty}$ est entière, s'annule aux points $q^{\mathbb{N}}$ et vérifie $(x ; p)_{\infty}=(1-x)(p x ; p)_{\infty}$. La fonction $\Theta_{q}(x)$ est holomorphe sur $\mathbb{C}^{*}$ et vérifie $\Theta_{q}(q x)=q x \Theta_{q}(x)$. Rappelons aussi la formule $\mathrm{du}$ triple produit de Jacobi

$$
\Theta_{q}(x)=(p ; p)_{\infty}(x ; p)_{\infty}\left(p x^{-1} ; p\right)_{\infty}
$$

qui montre que $\Theta_{q}(x)$ a pour ensemble de zéros $q^{\mathbb{Z}}$. Ces propriétés permettent d'établir la proposition qui suit.

Proposition 3. - Pour $(q, h) \in \mathcal{Q}^{+}$et $c \in \mathbb{C}$, la fonction $e_{c}^{(q, h)}(x)$ est solution de l'équation des caractères (3). Si $c=-s$ est un entier négatif, on a

$$
e_{-s}^{(q, h)}(x)=(-1)^{s} q^{-s} x^{-h^{[s]}} q .
$$

Si $c \in \mathbb{N}, e_{c}^{(q, h)}(x)$ est un polynôme de degré $c$. Enfin, si $c \notin \mathbb{N}$ et si $(q, h) \neq$ $(1,0)$, les pôles de $e_{c}^{(q, h)}(x)$ sont simples et situés aux points

(i) $h\left[c+\mathbb{N}^{*}\right]_{q}=\left\{h[c+n]_{q} ; n \in \mathbb{N}^{*}\right\}$ si $q>1$ et $h>0$,

(ii) $c+\mathbb{Z}$ si $q>1$ et $h=0$,

(iii) $h\left(c+\mathbb{N}^{*}\right)$ si $q=1$ et $h>0$.

Remarquons que ce choix de caractères se spécialise en celui de [3] lorsque $h=1$, celui de [8] lorsque $q=1$ et celui de [10] lorsque $h=0$, en remarquant dans ce dernier cas que la valeur propre est ici notée $q^{c}$. De plus, à constante près, lorsque $q \neq 1$ et $h \neq 0$, cette solution de (3) coïncide avec la solution canonique utilisée dans [10] pour résoudre l'équation aux $q$-différences en $t$ (régulière en 0 ) obtenue à partir de (3) après le changement de variable $t=1+(q-1) x / h$.

Ces fonctions permettent, comme on le verra plus bas, de résoudre les systèmes à matrice constante semi-simple. Pour traiter le cas général on s'appuie sur le résultat suivant qui s'obtient par dérivations successives par rapport à $c$ de (3), en convenant que, si $q=1,(\ln q /(q-1)) q^{-c}=1$ et, pour $j \geq 2$, $\left(\ln ^{j} q /(q-1)\right) q^{-c}=0$.

Proposition 4. - Pour tout $c \in \mathbb{C}$ et tout entier $k \geq 1$, les fonctions $\ell_{c, k}^{(q, h)}(x)$ vérifient la famille d'équations (4). 
Si $q=1$, la formule se réduit à la relation

$$
{ }_{1} \delta_{h} \ell_{c, k}^{(1, h)}(x)=c \ell_{c, k}^{(1, h)}(x)+\ell_{c, k-1}^{(1, h)}(x)
$$

et la famille de solutions choisie est celle utilisée dans [8]. La spécialisation $h=1$ correspond à l'une des deux familles utilisées dans [3] (celle notée $\widetilde{L}_{k}$ ). La spécialisation $h=0, q>1$ donne une famille différente de celle utilisée dans [10] et dont le lien avec cette autre famille «naturelle» est étudié dans [4]. Enfin la spécialisation $(q, h)=(1,0)$ correspond à la famille habituelle des logarithmes puisque

$$
\ell_{c, k}^{(1,0)}(x)=\frac{\ln ^{k}(-x)}{k !}(-x)^{c} .
$$

3.1.2. Solution canonique (cas constant). - Rappelons la convention $(\ln q /(q-1)) q^{-c}=1$ si $q=1$. Avec les notations de l'introduction, posons

$$
\widetilde{N}_{r_{j}}=\frac{q^{-c}}{1-q}\left(q^{-N_{r_{j}}}-I_{r_{j}}\right) .
$$

En remarquant que $q^{-N_{r}}-I_{r}=\sum_{j=1}^{r-1}(-1)^{i} \frac{\ln ^{j} q}{j !} N_{r}^{j}$, on déduit des formules (3) et (4) la proposition suivante dont la preuve est laissée au lecteur.

Proposition 5. - Pour tout $r \geq 1$, la matrice $r \times r$ :

$$
\mathcal{L}_{c, r}^{(q, h)}(x)=\left(\begin{array}{ccccc}
\ell_{c, 0}^{(q, h)}(x) & \ell_{c, 1}^{(q, h)}(x) & \ell_{c, 2}^{(q, h)}(x) & \cdots & \ell_{c, r-1}^{(q, h)}(x) \\
0 & \ell_{c, 0}^{(q, h)}(x) & \ell_{c, 1}^{(q, h)}(x) & \cdots & \ell_{c, r-2}^{(q, h)}(x) \\
\vdots & \ddots & \ddots & & \vdots \\
0 & \cdots & \cdots & 0 & \ell_{c, 0}^{(q, h)}(x)
\end{array}\right)
$$

est un système fondamental de solutions du système de dimension $r$ :

$$
{ }_{p} \delta_{h} Y(x)=A_{c, r}^{(q, h)} Y(x) .
$$

Reprenons les notations de la définition 3.

LEMME 2. - Toute matrice constante qui commute avec $J_{0}^{(q, h)}$ commute aussi avec $\mathcal{E}_{J_{0}^{(q, h)}}(x)$.

Démonstration. - Commençons par montrer que les matrices constantes qui commutent avec $J_{0}^{(q, h)}$ sont exactement celles qui commutent avec la forme de Jordan habituelle qui correspond à des blocs de la forme $-[-c]_{q} I_{r}+N_{r}$. Si $q=1$, les deux formes normales coïncident et il n'y a rien à prouver.

Si $q>1$, partageons une matrice $P$ qui commute avec $J_{0}^{(q, h)}$ en $P=$ $\left(P_{j k}\right)_{1 \leq j, k \leq m}$ où $P_{j k}$ est une matrice $r_{j} \times r_{k}$. Elle commute avec $J_{0}^{(q, h)}$ si et seulement si $P_{j k}=0$ lorsque $\left[-c_{j}\right]_{q} \neq\left[-c_{k}\right]_{q}$ et $\widetilde{N}_{r_{j}} P_{j k}=P_{j k} \widetilde{N}_{r_{k}}$ lorsque 
$\left[-c_{j}\right]_{q}=\left[-c_{k}\right]_{q}$, d'où aussi $\widetilde{N}_{r_{j}}^{i} P_{j k}=P_{j k} \widetilde{N}_{r_{k}}^{i}$, pour tout entier $i \geq 1$. Dans ce cas, si $c$ est tel que $q^{c}=q^{c_{j}}=q^{c_{k}}$, on déduit de (5) que

$$
N_{r_{j}}=-\frac{1}{\ln q} \ln \left(I_{r_{j}}-(q-1) q^{c} \widetilde{N}_{r_{j}}\right)=\frac{1}{\ln q} \sum_{i=1}^{r_{j}-1} \frac{(q-1)^{i} q^{i c}}{i} \widetilde{N}_{r_{j}}^{i}
$$

et donc aussi

$$
N_{r_{j}} P_{j k}=\frac{1}{\ln q} \sum_{i \geq 1} \frac{(q-1)^{i} q^{i c}}{i} \widetilde{N}_{r_{j}}^{i} P_{j k}=\frac{1}{\ln q} \sum_{i \geq 1} \frac{(q-1)^{i} q^{i c}}{i} P_{j k} \widetilde{N}_{r_{k}}^{i}=P_{j k} N_{r_{k}} .
$$

La matrice $\mathcal{E}_{J_{0}^{(q, h)}}(x)$ est diagonale par blocs correspondants à ceux de $J_{0}^{(q, h)}$, le $j$-ième bloc étant

$$
\mathcal{L}_{c_{j}, r_{j}}^{(q, h)}(x)=e_{c_{j}}^{(q, h)}(x) I_{r_{j}}+\sum_{i=1}^{r_{j}-1} \ell_{c_{j}, i}^{(q, h)}(x) N_{r_{j}}^{i}
$$

Elle commute avec $P$ si et seulement si on a $\mathcal{L}_{c_{j}, r_{j}}^{(q, h)}(x) P_{j k}=P_{j k} \mathcal{L}_{c_{k}, r_{k}}^{(q, h)}(x)$ pour tout $k, j$. Lorsque $\left[-c_{j}\right]_{q} \neq\left[-c_{k}\right]_{q}$, cette relation est claire puisque $P_{j k}=0$. Lorsque $\left[-c_{j}\right]_{q}=\left[-c_{k}\right]_{q}$, elle se déduit aisément des relations $N_{r_{j}} P_{j k}=P_{j k} N_{r_{k}}$.

Soit $A_{0}^{(q, h)}$ la matrice du système (1). Puisqu'elle est inversible, on peut supposer que ses valeurs propres sont écrites sous la forme $-\left[-c_{1}\right]_{q}, \ldots,-\left[-c_{n}\right]_{q}$ (répétées s'il y a plusieurs blocs de Jordan pour une même valeur propre) et qu'elles vérifient la condition de non résonnance qui s'écrit maintenant $c_{i}-c_{j} \notin \mathbb{Z}^{*}$. La relation (6) permet de prendre $J_{0}^{(q, h)}$ pour forme normale de $A_{0}^{(q, h)}$, c'est-à-dire de supposer qu'il existe une matrice $P \in \mathrm{Gl}_{n}(\mathbb{C})$ telle que $A_{0}^{(q, h)}=P J_{0}^{(q, h)} P^{-1}$. La matrice

$$
P \mathcal{E}_{J_{0}^{(q, h)}}(x) P^{-1}
$$

est clairement un système fondamental de solutions du système défini par $A_{0}^{(q, h)}$. Voyons ce qu'il en est de la dépendance de ce système fondamental par rapport à la matrice de passage $P$.

Lemme 3. - Si $P_{1}$ et $P_{2}$ sont deux matrices conjuguant $A_{0}^{(q, h)} \grave{a} J_{0}^{(q, h)}$, alors

$$
P_{1} \mathcal{E}_{J_{0}^{(q, h)}}(x) P_{1}^{-1}=P_{2} \mathcal{E}_{J_{0}^{(q, h)}}(x) P_{2}^{-1} .
$$

Démonstration. - L'hypothèse implique que $P_{1}^{-1} P_{2}$ commute avec $J_{0}^{(q, h)}$ et donc, d'après le lemme 2 ,

$$
P_{1}^{-1} P_{2} \mathcal{E}_{J_{0}^{(q, h)}}(x)=\mathcal{E}_{J_{0}^{(q, h)}}(x) P_{1}^{-1} P_{2}
$$

ou encore $P_{1} \mathcal{E}_{J_{0}^{(q, h)}}(x) P_{1}^{-1}=P_{2} \mathcal{E}_{J_{0}^{(q, h)}}(x) P_{2}^{-1}$. 
Cela montre que $\mathcal{E}_{A_{0}^{(q, h)}}(x)$ est effectivement indépendante des matrices conjuguantes choisies et justifie la définition 3 : dans le cas d'un système de matrice constante, on appelle solution canonique la matrice $\mathcal{E}_{A_{0}^{(q, h)}}(x)$.

3.2. Transformation de jauge. - Nous allons maintenant établir l'existence d'une (unique) famille de transformations de jauge tangentes à l'identité ramenant la famille de systèmes (1) à une famille de systèmes à coefficients constants, ce dernier cas ayant été traité dans la section précédente. Cela se réalise en plusieurs étapes et le théorème qui suit est un outil essentiel.

THÉORÈme 3. - Soit $\Lambda$ une partie bornée de $\mathcal{Q}^{+}$. Considérons une famille (1) fuchsienne non résonnante $(C, \lambda)$ sur $\Lambda$. On note $C_{0}^{(q, h)}$ l'inverse de la matrice $I+(1-q) A_{0}^{(q, h)}$ et on suppose qu'il existe $\alpha>0$ tel que $\left\|C_{0}^{(q, h)}\right\| \leq \alpha$, pour tout $(q, h) \in \Lambda$. La non résonance implique que, pour tout $s \geq 1$, l'opérateur défini sur $M_{n}(\mathbb{C})$ par

$$
U \longmapsto\left(A_{0}^{(q, h)}+[s]_{q} I\right) U-q^{s} U A_{0}^{(q, h)}
$$

est inversible. On note $\widetilde{L}_{s}^{(q, h)}$ son inverse et on suppose qu'il existe $M>0$ tel que pour tout $s \geq 1$ et tout $(q, h) \in \Lambda$, l'opérateur $\widetilde{L}_{s}^{(q, h)}$ soit de norme inférieure ou égale à $M$.

Alors, pour chaque $(q, h) \in \Lambda$, il existe une unique transformation de jauge $F^{(q, h)}(x)$ développable en série de $(q, h)$-factorielles, tangente à l'identité, qui transforme le système précédent en celui de matrice constante $A_{0}^{(q, h)}$; autrement dit

$$
\left(A^{(q, h)}\right)^{F^{(q, h)}}=A_{0}^{(q, h)} .
$$

De plus, il existe $C^{\prime}>0$ et $\lambda^{\prime}>0$ tels que la famille $\left(F^{(q, h)}(x)\right)_{(q, h) \in \Lambda}$ est de type $\left(C^{\prime}, \lambda^{\prime}\right)$.

Le cas $h=0$ et $q=1$ est le classique théorème concernant les systèmes différentiels fuchsiens. Les trois spécialisations $h=1$ ou $q=1$ (et $h>0$ ) ou $h=0$ (et $q>1$ ) donnent les résultats établis dans [3], [8] ou [10] et la preuve qui suit reprend la même démarche.

3.2.1. Un résultat préliminaire. - La preuve du théorème 1 repose sur la proposition suivante qui établit l'existence d'un vecteur solution du système (1) lorsqu'on en fixe convenablement la valeur à l'infini.

Proposition 6. - Soit une famille (1) fuchsienne $(C, \lambda)$ sur $\Lambda$. On suppose que, pour tout $s \geq 1,-[s]_{q}$ n'est pas valeur propre de la matrice $A_{0}^{(q, h)}$. Soit $U_{0}$ un vecteur non nul de $\mathbb{C}^{n}$. Pour tout $(q, h) \in \Lambda$, le système (1) a une solution qui est une série (formelle) de $(q, h)$-factorielles de terme constant $U_{0}$ si et seulement si $U_{0}$ appartient au noyau de $A_{0}^{(q, h)}$. Une telle solution est alors 
unique. De plus, si $b=\sup _{s \geq 1,(q, h) \in \Lambda}\left\|\left([s]_{q} I+A_{0}^{(q, h)}\right)^{-1}\right\|$ est fini, la famille formée par ces solutions est de type $\left(C b\left|U_{0}\right|, C b+\lambda\right)$.

Démonstration. - Notons $A^{(q, h)}(x)=\sum_{s=0}^{+\infty} A_{s}^{(q, h)} x^{-{ }^{[s]} q}$ et cherchons $Y^{(q, h)}(x)$ sous la forme

$$
Y^{(q, h)}(x)=U_{0}+\sum_{s=1}^{+\infty} Y_{s}^{(q, h)} x^{-{ }_{h}^{[s]}} q
$$

Partie formelle. - D'après la formule du produit, on a

$$
A^{(q, h)}(x) Y^{(q, h)}(x)=\sum_{s=0}^{+\infty} C_{s}^{(q, h)} x^{-{ }_{h}[s]} q
$$

avec $C_{0}^{(q, h)}=A_{0}^{(q, h)} U_{0}$ et, pour $s \geq 1$

$$
C_{s}^{(q, h)}=A_{0}^{(q, h)} Y_{s}^{(q, h)}+A_{s}^{(q, h)} U_{0}+\sum_{(i, j, k) \in J_{s}} h^{k} q^{k+i j} c_{i, j, k} A_{i}^{(q, h)} Y_{j}^{(q, h)} .
$$

D'autre part, puisque

$$
{ }_{p} \delta_{h} Y^{(q, h)}(x)=\sum_{s=1}^{+\infty}-[s]_{q} Y_{s}^{(q, h)} x^{-{ }_{h^{[s]}} q}
$$

$Y^{(q, h)}$ est solution de (1) si et seulement si ses coefficients vérifient $A_{0}^{(q, h)} U_{0}=0$ et

$$
-[s]_{q} Y_{s}^{(q, h}=A_{0}^{(q, h)} Y_{s}^{(q, h)}+A_{s}^{(q, h)} U_{0}+\sum_{(i, j, k) \in J_{s}} h^{k} q^{k+i j} c_{i, j, k} A_{i}^{(q, h)} Y_{j}^{(q, h)}
$$

ou encore $A_{0}^{(q, h)} U_{0}=0$ et

$$
-\left([s]_{q} I+A_{0}^{(q, h)}\right) Y_{s}^{(q, h)}=A_{s}^{(q, h)} U_{0}+\sum_{(i, j, k) \in J_{s}} h^{k} q^{k+i j} c_{i, j, k} A_{i}^{(q, h)} Y_{j}^{(q, h)} .
$$

Il est donc nécessaire que $U_{0}$ soit dans $\operatorname{ker} A_{0}^{(q, h)}$. Comme, par hypothèse, la matrice $[s]_{q} I+A_{0}^{(q, h)}$ est inversible, le système se résout de proche en proche :

$$
Y_{s}^{(q, h)}=-\left([s]_{q} I+A_{0}^{(q, h)}\right)^{-1}\left[A_{s}^{(q, h)} U_{0}+\sum_{(i, j, k) \in J_{s}} h^{k} q^{k+i j} c_{i, j, k} A_{i}^{(q, h)} Y_{j}^{(q, h)}\right] .
$$

Ceci termine l'aspect formel de la proposition.

Partie convergente. - Établissons maintenant, sous l'hypothèse indiquée, la convergence de cette solution. Notons

$$
a_{s}^{(q, h)}=\left\|A_{s}^{(q, h)}\right\|, \quad u_{0}=\left|U_{0}\right|, \quad y_{s}^{(q, h)}=\left|Y_{s}^{(q, h)}\right| \text { pour tout } s \geq 1 .
$$


On déduit de l'équation récurrente ci-dessus et de la définition de $b$, pour $s \geq 1$, les majorations

$$
y_{s}^{(q, h)} \leq b\left[a_{s}^{(q, h)} u_{0}+\sum_{(i, j, k) \in J_{s}} h^{k} q^{k+i j} c_{i, j, k} a_{i}^{(q, h)} y_{j}^{(q, h)}\right] .
$$

Introduisons la suite définie récursivement par

$$
z_{1}^{(q, h)}=b a_{1}^{(q, h)} u_{0} \quad \text { et } \quad z_{s}^{(q, h)}=b\left[a_{s}^{(q, h)} u_{0}+\sum_{(i, j, k) \in J_{s}} q^{k+i j} h^{k} c_{i, j, k} a_{i}^{(q, h)} z_{j}^{(q, h)}\right]
$$

de sorte que $y_{s}^{(q, h)} \leq z_{s}^{(q, h)}$, pour tout $s \geq 1$. Notons

$$
z^{(q, h)}(x)=\sum_{s=1}^{+\infty} z_{s}^{(q, h)} x^{-{ }_{h}^{[s]}} q \quad \text { et } \quad a^{(q, h)}(x)=\sum_{s=1}^{+\infty} a_{s}^{(q, h)} x^{-{ }_{h}[s]} q .
$$

La relation

$$
z^{(q, h)}(x)=b\left(u_{0} a^{(q, h)}(x)+a^{(q, h)}(x) z^{(q, h)}(x)\right) .
$$

est une conséquence immédiate de la récurrence définissant $z^{(q, h)}(x)$. On en déduit

$$
z^{(q, h)}(x)=\frac{b u_{0} a^{(q, h)}(x)}{1-b a^{(q, h)}(x)}=-u_{0}\left(1-\frac{1}{1-b a^{(q, h)}(x)}\right)
$$

et il ne reste qu'à appliquer la proposition 2 pour obtenir

$$
z_{s}^{(q, h)} \leq C b u_{0} q^{s-1}\left((\lambda+C b)^{-h^{[s-1]}} q\right)^{-1}
$$

Lorsque $A_{0}^{(q, h)}=0$, le système est « régulier ». En appliquant le résultat précédent à chacun des vecteurs de la base canonique $\left(\varepsilon_{1}, \ldots, \varepsilon_{n}\right)$ de $\mathbb{C}^{n}$, on obtient dans ce cas un système fondamental de solutions tangent à l'identité :

Corollaire 1. - Soit

$$
{ }_{p} \delta_{h} Y(x)=A^{(q, h)} Y(x)
$$

une famille de systèmes réguliers, de type $(C, \lambda)$ sur $\Lambda$. Ces équations admettent chacune un système fondamental de solutions, tangent à l'identité, formé de séries de $(q, h)$-factorielles. La famille ainsi définie est de type $\left(C^{\prime}, C+\lambda\right)$ avec $C^{\prime}=C \max _{1 \leq i \leq n}\left|\varepsilon_{i}\right|$. 
3.2.2. Preuve du théorème 3. - Notons $F^{(q, h)}(x)=I+\sum_{s=1}^{+\infty} F_{s}^{(q, h)} x^{-{ }_{h}[s]} q$ la transformation de jauge cherchée. La condition qu'elle doit vérifier s'écrit

$$
A^{(q, h)}(x) F^{(q, h)}(x)-{ }_{p} \delta_{h} F^{(q, h)}(x)=F^{(q, h)}(p x-p h) A_{0}^{(q, h)}
$$

ou encore

${ }_{p} \delta_{h} F^{(q, h)}(x)\left[I+\frac{(p-1) x-p h}{p(x-h)} A_{0}^{(q, h)}\right]=\left[A^{(q, h)}(x) F^{(q, h)}(x)-F^{(q, h)}(x) A_{0}^{(q, h)}\right]$.

D'autre part, si $a \in \mathbb{C}$ est tel que $b=1+(1-q) a \neq 0$, la formule (2) permet d'écrire, en posant $d=a / b$ et $\mu=(1+a) h / b=(1+q d) h$,

$$
\begin{gathered}
{\left[1+\frac{(p-1) x-p h}{p(x-h)} a\right]^{-1}=\frac{x-h}{b x-(1+a) h}=\frac{1}{b}\left[1+\frac{q a h}{b x-(1+a) h}\right]} \\
=\frac{1}{b}\left[1+\frac{q d h}{x-\mu}\right]=\frac{1}{b}\left[1+q d h \sum_{s \geq 1} q^{s-1}\left(\mu^{-{ }_{h}[s-1]_{q}}\right)^{-1} x^{-{ }^{[s]}} q\right] \\
=\frac{1}{b}\left[1+\sum_{s \geq 1} q^{s} h^{s}\left(d^{-[s]_{q}}\right)^{-1} x^{-h^{[s]}} q .\right.
\end{gathered}
$$

Cette dernière égalité s'obtient en remarquant que, puisque $\mu=(1+q d) h$, on a $\left.d h\left(\mu^{-h^{[s-1]}} q\right)\right)^{-1}=h^{s}\left(d^{-[s]_{q}}\right)^{-1}$.

Dans ces expressions, qui sont des polynômes en $d$, on peut remplacer le nombre complexe $d$ par la matrice

$$
D_{0}^{(q, h)}=C_{0}^{(q, h)} A_{0}^{(q, h)},
$$

et donc $a$ par $A_{0}^{(q, h)}$ et $1 / b$ par $C_{0}^{(q, h)}$. On obtient le développement

$$
\left[I+\frac{(p-1) x-p h}{p(x-h)} A_{0}^{(q, h)}\right]^{-1}=\sum_{s=0}^{+\infty} C_{s}^{(q, h)} x^{-{ }_{h}[s]} q
$$

avec, pour $s \geq 1$,

$$
C_{s}^{(q, h)}=h^{s} q^{s} C_{0}^{(q, h)} \prod_{k=0}^{s-1}\left(q^{k} D_{0}^{(q, h)}+[k]_{q} I\right) .
$$

L'équation (7) peut se mettre sous la forme

${ }_{p} \delta_{h} F^{(q, h)}(x)=\left[A^{(q, h)}(x) F(x)^{(q, h)}-F^{(q, h)}(x) A_{0}^{(q, h)}\right] \cdot\left[I+\frac{(p-1) x-p h}{p(x-h)} A_{0}^{(q, h)}\right]^{-1}$

et s'interpréter comme un système aux $(q, h)$-différences de dimension $n^{2}$ auquel on peut appliquer la proposition 6 . En effet :

$$
{ }_{p} \delta_{h} F^{(q, h)}(x)=\sum_{s=0}^{+\infty} L_{s}^{(q, h)}\left(F^{(q, h)}(x)\right) x^{-{ }_{h}[s]} q
$$

TOME $136-2008-\mathrm{N}^{\mathrm{O}} 1$ 
où $L_{s}^{(q, h)}$ est l'opérateur linéaire agissant sur $M_{n}(\mathbb{C})$ par

$$
L_{0}^{(q, h)}(U)=\left[A_{0}^{(q, h)} U-U A_{0}^{(q, h)}\right] C_{0}^{(q, h)},
$$

et, pour $s \geq 1$,

$$
\begin{gathered}
L_{s}^{(q, h)}(U)=A_{s}^{(q, h)} U C_{0}^{(q, h)}+\left(A_{0}^{(q, h)} U-U A_{0}^{(q, h)}\right) C_{s}^{(q, h)} \\
+\sum_{(i, j, k) \in J_{s}} h^{k} q^{k+i j} c_{i, j, k} A_{i}^{(q, h)} U C_{j}^{(q, h)} .
\end{gathered}
$$

L'opérateur linéaire $L_{0}^{(q, h)}$ admet 0 pour valeur propre et $I$ est un vecteur propre associé. Pour tout $s \geq 1,-[s]_{q}$ n'est pas valeur propre de $L_{0}^{(q, h)}$ : cela résulte de l'hypothèse de non résonance puisque l'égalité $L_{0}^{(q, h)}(U)=-[s]_{q} U$ équivaut à

$$
A_{0}^{(q, h)} U-U A_{0}^{(q, h)}=-[s]_{q} U\left(I+(1-q) A_{0}^{(q, h)}\right)=-[s]_{q} U+\left(q^{s}-1\right) U A_{0}^{(q, h)}
$$

et donc aussi à

$$
\left(A_{0}^{(q, h)}+[s]_{q} I\right) U-q^{s} U A_{0}^{(q, h)}=0 .
$$

De plus l'hypothèse faite permet de majorer uniformément sur $\Lambda$ la norme de l'opérateur $\left(L_{0}^{(q, h)}+[s]_{q} \mathrm{Id}\right)^{-1}$ qui s'exprime en fonction de l'opérateur $\widetilde{L}_{s}^{(q, h)}$ et de la matrice $I+(1-q) A_{0}^{(q, h)}$ dont la norme est bornée par hypothèse. Ensuite, puisque $\left\|D_{0}^{(q, h)}\right\| \leq C \alpha$, on déduit de la formule donnant $C_{s}^{(q, h)}$ la majoration

$$
\left\|C_{s}^{(q, h)}\right\| \leq \alpha h^{s} q^{s} \prod_{k=0}^{s-1}\left(q^{k} C \alpha+[k]_{q}\right)=\alpha h^{s} q^{s}\left((C \alpha)^{-[s]_{q}}\right)^{-1} .
$$

D'où l'estimation suivante pour la norme de $L_{s}^{(q, h)}$ :

$$
\begin{aligned}
\left\|L_{s}^{(q, h)}\right\| \leq C \alpha & q^{s-1}\left(\lambda^{-} h^{[s-1]} q\right)^{-1}+2 C \alpha h^{s} q^{s}\left((C \alpha)^{-[s]_{q}}\right)^{-1} \\
& +C \sum_{(i, j, k) \in J_{s}} h^{k+j} q^{i j+s-1} c_{i, j, k}\left(\lambda^{-} h^{[i-1]_{q}}\right)^{-1}\left((C \alpha)^{-[j]_{q}}\right)^{-1} .
\end{aligned}
$$

En utilisant la formule du produit, le développement (2) et la formule écrite plus haut avec $\mu=(1+q C \alpha) h$, on voit que la série $\sum_{s \geq 0}\left\|L_{s}^{(q, h)}\right\| x^{-h^{[s]}} q$ admet pour série majorante la série qui constitue le développement en série de $(q, h)$ factorielles de la fonction

$$
C \alpha\left(2+\frac{1}{x-\lambda}\right)\left(1+\frac{q C \alpha h}{x-\mu}\right) .
$$

Il s'ensuit que la famille $L_{s}^{(q, h)}$ est fuchsienne $\left(C^{\prime \prime}, \lambda^{\prime \prime}\right)$ avec

$$
\lambda^{\prime \prime}=\max (\lambda,(1+B C \alpha) B)
$$

si $B$ est un majorant de $\Lambda$. Nous sommes donc en mesure d'appliquer la proposition 6 et cela termine la démonstration du théorème 3 . 
3.3. Fin de la preuve du théorème 1 . - La preuve du théorème 1 se fait maintenant de la manière suivante. On a déjà remarqué que la condition $c_{i}-c_{j} \notin \mathbb{Z}$ assure la non résonance. Un calcul facile montre ensuite que la matrice $C_{0}^{(q, h)}$ est conjuguée à la matrice $\operatorname{diag}\left(q^{c_{1} I_{r_{1}}+N_{r_{1}}}, \ldots, q^{c_{m} I_{r_{m}}+N_{r_{m}}}\right)$ et la première hypothèse du théorème 3 est satisfaite dès qu'il existe une famille bornée de conjugaisons. L'opérateur $\widetilde{L}_{s}^{(q, h)}$ est l'inverse d'un opérateur qui s'écrit (toujours à conjugaison près) $U \mapsto \operatorname{diag}\left(B_{1}, \ldots, B_{m}\right)$ où $B_{j}=q^{-c_{j}}[s]_{q} U+\widetilde{N}_{r_{j}} U-q^{s} U \widetilde{N}_{r_{j}}$ et la deuxième hypothèse découle $\mathrm{du}$ fait que $[s]_{q}$ et $q^{s}$ tendent vers l'infini avec $s$. Les autres affirmations du théorème sont conséquences des résultats du paragraphe précédent et de la remarque suivante. Si $F^{(q, h)}(x) P \mathcal{E}_{J_{0}^{(q, h)}}(x)$ est solution de (1), alors

$$
A^{(q, h)^{F^{(q, h)}(x) P}}=J_{0}^{(q, h)}
$$

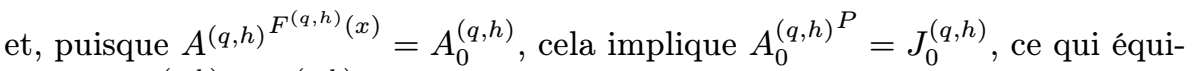
vaut à $P J_{0}^{(q, h)}=A_{0}^{(q, h)} P$.

\section{Preuve du théorème 2}

Il s'agit ici d'étudier la continuité en $(q, h)$ de la solution canonique obtenue au théorème 1 lorsqu'on suppose que la matrice $A^{(q, h)}(x)$ est continue (en un sens à préciser) en un point $\left(q_{0}, h_{0}\right)$. Nous traitons ce problème lorsque $\left(q_{0}, h_{0}\right)$ est un point de la frontière de $\mathcal{Q}^{+}$, c'est-à-dire lorsque $q_{0}=1$ ou $h_{0}=0$. Dans ce cas il est habituel de parler plutôt de confluence.

La forme de la solution canonique indique qu'on peut ici aussi séparer le problème en deux parties que nous aborderons successivement : la confluence de la transformation de jauge d'une part et celle de la partie «log-car » provenant de la matrice constante d'autre part.

4.1. Confluence de la partie log-car. - La famille des « monômes » $\left(x^{-{ }^{[}}{ }^{[s]} q\right)$, qui sont les caractères correspondant à $c$ entier négatif, est continue sur $\mathcal{Q}^{+}$. C'est également le cas de la famille de polynômes $\left(e_{c}^{(q, h)}(x)\right)$ où $c \in \mathbb{N}$. Par contre, lorsque $c$ n'est pas entier, nous verrons que la famille des caractères choisis et des logarithmes associés n'a pas de limite en certains points du bord de $\mathcal{Q}^{+}$. On rencontrera cette situation dans le cas $\mathrm{C} 1$; en revanche, on énoncera une propriété de «normalité » et on déterminera l'ensemble des valeurs d'adhérence. L'absence de limite n'est pas un phénomène surprenant car la définition de $e_{c}^{(q, h)}(x)$ pour $q>1, h>0$ provient du cas $h=0$ par l'utilisation du changement de variable homographique $t=(q-1) x / h+1$. Ce changement de variable n'a pas de limite (qui soit un changement de variable) en un point 
du bord de $\mathcal{Q}^{+}$autre que $(1,0)$. Lorsque $\left(q_{0}, h_{0}\right)=(1,0)$, il faut supposer que $(q-1) / h$ a une limite si l'on veut que le changement de variable en ait une.

Signalons un autre problème. L'équation $\sigma_{q} y(x)=q^{c} y(x)$ admet aussi la solution, indépendante de $q$, mais ramifiée : $x^{c}$. La fonction $q$-périodique qui relie les deux caractères s'exprime à l'aide de la fonction $K(x, t)$ introduite par De Sole et Kac dans [1]. Cette remarque est l'objet du lemme ci-dessous. Nous reprenons les notations suivantes de [1]. Pour $a, t \in \mathbb{C}$, on note

$$
(1+a)_{p}^{t}=\frac{(-a ; p)_{\infty}}{\left(-p^{t} a ; p\right)_{\infty}} .
$$

Si $t \in \mathbb{C}$ et si on a choisi une détermination du logarithme de $x \neq 0$, on pose

$$
K(x, t)=x^{t}\left(1+\frac{1}{x}\right)_{p}^{t}(1+p x)_{p}^{-t} .
$$

Cette fonction vérifie les propriétés suivantes :

1) $K(x, t+1)=p^{t} K(x, t)$;

2) $K(q x, t)=K(x, t)$ si l'on suppose $\arg (q x)=\arg x$; autrement dit, comme fonction de $x, K(x, t)$ est une $q$-constante;

3) $K(x, t)=K(1 / x, 1-t)$ si l'on impose $\arg 1 / x=-\arg x$ de sorte que $(1 / x)^{t}=x^{-t}$

4) si $n \in \mathbb{Z}, K(x, n)=p^{\frac{1}{2} n(n-1)}$ et est donc indépendant de $x$.

Lemme 4. - Pour $q>1$, on a

$$
e_{c}^{(q, 0)}(x)=(-x)^{c} K\left(-\frac{1}{x}, c\right)=(-x)^{c} K(-x, 1-c) .
$$

Démonstration. - Par définition, $e_{c}^{(q, 0)}(x)=\Theta_{q}(x) / \Theta_{q, q^{c}}(x)$ et en utilisant la formule du triple produit :

$$
\begin{aligned}
e_{c}^{(q, 0)}(x) & =\frac{(x ; p)_{\infty}(p / x ; p)_{\infty}}{\left(p^{c} x ; p\right)_{\infty}\left(p^{1-c} / x ; p\right)_{\infty}} \\
& =(1-x)_{p}^{c}\left(1-\frac{p}{x}\right)_{p}^{-c}=(-x)^{c} K\left(-\frac{1}{x}, c\right)=(-x)^{c} K(-x, 1-c) .
\end{aligned}
$$

Avec cette notation, pour $q>1$ et $h>0$ on a, en posant $z=(1-p) x / h+p$,

$$
e_{c}^{(q, h)}(x)=\left(\frac{h}{1-p}\right)^{c}(1-z)_{p}^{c} .
$$


4.1.1. Confluence des caractères. - On s'intéresse dans ce paragraphe au problème suivant. Soit $\left(q_{0}, h_{0}\right)$ un point du bord de $\mathcal{Q}^{+}$. La fonction $e_{c}^{(q, h)}(x)$ tend-elle vers la fonction $e_{c}^{\left(q_{0}, h_{0}\right)}(x)$ lorsque $(q, h)$ tend vers $\left(q_{0}, h_{0}\right)$ ?

Constatons tout d'abord que la réponse est négative lorsque $q_{0}>1$ et $h_{0}=0$. Reprenons les notations précédentes et définissons, pour $p=1 / q$ fixé, le réel $\xi$ par la condition $h=(1-p) p^{\xi}$, de sorte que $h \rightarrow 0$ si et seulement si $\xi \rightarrow+\infty$. On a

$$
\begin{aligned}
e_{c}^{(q, h)}(x) & =p^{\xi c}\left(1-p^{-\xi} x-p\right)_{p}^{c}=p^{\xi c}\left(1-p^{-\xi}\left(x-p^{1+\xi}\right)\right)_{p}^{c} \\
& =p^{\xi c} \frac{K\left(-p^{\xi} /\left(x-p^{1+\xi}\right), c\right)}{\left(1-p^{\xi+1} /\left(x-p^{1+\xi}\right)\right)_{p}^{-c}}\left(-p^{-\xi}\left(x-p^{1+\xi}\right)\right)^{c} \\
& =\frac{K\left(-p^{\xi} /\left(x-p^{1+\xi}\right), c\right)}{\left(1-p^{\xi+1} /\left(x-p^{1+\xi}\right)\right)_{p}^{-c}}\left(-\left(x-p^{1+\xi}\right)\right)^{c} \\
& =\frac{K\left(-p^{\{\xi\}} /\left(x-p^{1+\xi}\right), c\right)}{\left(1-p^{\xi+1} /\left(x-p^{1+\xi}\right)\right)_{p}^{-c}}\left(-\left(x-p^{1+\xi}\right)\right)^{c}
\end{aligned}
$$

en désignant par $\{\xi\} \in[0,1$ [ la partie fractionnaire de $\xi$. En utilisant le fait que $p^{\xi} \rightarrow 0$ quand $\xi \rightarrow+\infty$, on voit que le dénominateur tend vers 1 et que le facteur $\left(-\left(x-p^{1+\xi}\right)\right)^{c}$ tend vers le caractère $(-x)^{c}$. Une condition nécessaire à l'existence d'une limite est alors que $\xi \rightarrow+\infty$ de sorte que $\{\xi\}$ ait une limite. Or, même dans ce cas le facteur $K\left(-p^{-\{\xi\}} / x, c\right)$ est $q$-constant mais non constant. Il n'aurait donc pas été plus intéressant de faire un autre choix de caractères pour les opérateurs aux $q$-différences.

En utilisant le lemme 4, il vient

$$
e_{c}^{(q, h)}(x)=\frac{\left(-p^{-\{\xi\}}\left(x-p^{1+\xi}\right)\right)^{-c}}{\left(1-p^{\xi+1} /\left(x-p^{1+\xi}\right)\right)_{p}^{-c}}\left(-\left(x-p^{1+\xi}\right)\right)^{c} e_{c}^{(q, 0)}\left(p^{-\{\xi\}}\left(x-p^{1+\xi}\right)\right)
$$

et donc

$$
e_{c}^{(q, h)}(x)=\frac{p^{\{\xi\} c}}{\left(1-p^{\xi+1} /\left(x-p^{1+\xi}\right)\right)_{p}^{-c}} e_{c}^{(q, 0)}\left(p^{-\{\xi\}}\left(x-p^{1+\xi}\right)\right) .
$$

Cette expression montre qu'il n'y a pas de limite si $\xi \rightarrow+\infty$ mais que, si $\xi=\xi_{0}+n$ avec $\xi_{0}$ fixé dans [0,1[ (ou si, plus généralement, $\left(\xi_{n}\right)_{n \geq 1}$ est une suite telle que $\left\{\xi_{n}\right\}$ tend vers $\left\{\xi_{0}\right\}$ lorsque $n$ tend vers l'infini) il y a une limite quand $n \rightarrow+\infty$ qui vaut

$$
p^{\xi_{0} c} e_{c}^{(q, 0)}\left(p^{-\xi_{0}} x\right)=p^{\xi_{0} c} \frac{e_{c-\xi_{0}}^{(q, 0)}(x)}{e_{-\xi_{0}}^{(q, 0)}(x)}
$$

Le même calcul montre aussi l'absence de limite dans le cas C3 sous l'hypothèse $(\mathrm{H})$ avec $\ell=0$. 
Ces propriétés «négatives » donnent de l'intérêt au résultat de normalité qui figure dans le théorème suivant. Nous commençons par énoncer un lemme dont la preuve se trouve dans [10], p. 1045.

Lemme 5. - Soient $p$ un réel tel que $0<p<1, a \in \mathbb{C}, b \in \mathbb{C} \backslash p^{-\mathbb{N}}$. On pose $d=|a-b|$ et $\eta=\inf _{s \in \mathbb{N}}\left|1-p^{s} b\right|$. Alors, pour tout $s \in \mathbb{N}$,

$$
\left|\frac{(a ; p)_{s}}{(b ; p)_{s}}\right| \leq \exp \left(\frac{d}{\eta} \frac{1}{1-p}\right)
$$

THÉORÈME 4. -

$\mathrm{C} 1$ : La famille $\left(e_{c}^{\left(q_{0}, h\right)}(x)\right)_{0<h \leq \varepsilon}$ est normale sur $\mathbb{C} \backslash q_{0}^{c} \mathbb{R}^{+}$pour tout $\varepsilon>0$. Si $\xi \in\left[0,1\left[\right.\right.$ la suite $\left(e_{c}^{\left(q_{0}, h_{n}\right)}\right)_{n \geq 0}$ où $h_{n}=\left(1-p_{0}\right) p_{0}^{\xi+n}$ $\left(p_{0}=1 / q_{0}\right)$ tend vers

$$
\tilde{e}_{c}^{\left(q_{0}, \xi\right)}(x)=p_{0}^{\xi c} \frac{\Theta_{q_{0}, p_{0}^{\xi}}}{\Theta_{q_{0}, p_{0}^{\xi} p_{0}^{-c}}}=p_{0}^{\xi c} \frac{e_{c-\xi}^{\left(q_{0}, 0\right)}(x)}{e_{-\xi}^{\left(q_{0}, 0\right)}(x)}
$$

uniformément sur tout compact de $\mathbb{C} \backslash q_{0}^{c} \mathbb{R}^{+}$. Réciproquement si la suite $\left(e_{c}^{\left(q_{0}, h_{n}\right)}(x)\right)_{n \geq 0}$ converge lorsque $h_{n} \rightarrow 0$, il existe $\xi \in[0,1[$ tel que sa limite soit $\tilde{e}_{c}^{\left(q_{0}, \xi\right)}(x)$.

$\mathrm{C} 2:$ Pour tout $c \in \mathbb{C}$, la famille $\left(e_{c}^{(q, h)}\right)_{(q, h) \in \mathcal{Q}}$ converge uniformémement sur tout compact de $\mathbb{C} \backslash\left(c+h_{0} \mathbb{N}^{*}\right)$ vers $e_{c}^{\left(1, h_{0}\right)}$.

C3: On suppose $\ell \neq 0$. Pour tout $c \in \mathbb{C}$, la famille $\left(e_{c}^{(q, h)}\right)_{(q, h) \in \mathcal{Q}^{*}}$ converge uniformémement sur tout compact de $\mathbb{C} \backslash \mathbb{R}^{+}$vers $e_{c}$.

\section{Démonstration}

C1. - Rappelons l'égalité

$$
e_{c}^{\left(q_{0}, h\right)}(x)=\frac{K\left(-p_{0}^{\{\xi\}} /\left(x-p_{0}^{1+\xi}\right), c\right)}{\left(1-p_{0}^{\xi+1} /\left(x-p_{0}^{1+\xi}\right)\right)_{p_{0}}^{-c}}\left(-\left(x-p_{0}^{1+\xi}\right)\right)^{c}
$$

où le réel $\xi$ est défini par $h=\left(1-p_{0}\right) p_{0}^{\xi}$. Soit $K$ un compact de $\mathbb{C} \backslash q_{0}^{c} \mathbb{R}^{+}$. Puisque $\xi \rightarrow+\infty$ lorsque $h \rightarrow 0$, il existe $A_{1}>0$ tel que, pour tout $\xi \geq A_{1}$ et pour tout $x \in K,\left|p_{0}^{\xi+1} /\left(x-p_{0}^{1+\xi}\right)\right| \leq \frac{1}{2}$. On en déduit qu'il existe $M_{1}>0$ tel que pour tout $\xi \geq A_{1}$ et pour tout $x \in K, 1 /\left|\left(1-p_{0}^{\xi+1} /\left(x-p_{0}^{1+\xi}\right)\right)_{p_{0}}^{-c}\right| \leq M_{1}$. D'autre part, puisque $K$ est un compact de $\mathbb{C} \backslash q_{0}^{c} \mathbb{R}^{+}$et que $p_{0}^{\xi}$ tend vers 0 avec $h$, il existe $A_{2}>0$ et $\theta \in$ ] $0, \pi$ [ tels que pour tout $\xi \geq A_{2}$ et pour tout $x \in K,\left|\arg \left(p_{0}^{\{\xi\}} /\left(x-p_{0}^{1+\xi}\right) q_{0}^{c}\right)\right| \geq \theta$. Ainsi, puisque $p_{0} \leq p_{0}^{\{\xi\}} \leq 1$, il existe un compact $K^{\prime}$ de $\mathbb{C} \backslash q_{0}^{c} \mathbb{R}^{+}$contenant $p_{0}^{\{\xi\}} /\left(x-p_{0}^{1+\xi}\right)$ pour tout $\xi \geq A_{2}$ et pour tout $x \in K$. Il existe donc $M_{2}>0$ tel que pour tout $\xi \geq A_{2}$ et tout $x \in K,\left|K\left(-p_{0}^{\{\xi\}} /\left(x-p_{0}^{1+\xi}\right), c\right)\right| \leq M_{2}$. On en déduit que la famille des 
fonctions $e_{c}^{\left(q_{0}, h\right)}, h>0$ petit, est uniformément bornée sur $K$. Cela prouve la normalité.

Les deux autres assertions sont des conséquences immédiates de la formule (8). Pour la réciproque, il suffit d'utiliser le fait que la suite des $\left\{\xi_{n}\right\}$ admet toujours au moins une valeur d'adhérence.

C2. - Le cas $h=h_{0}=1$ est traité dans [3]. On emploie la même méthode. On fait le changement de variable $x=h[u]_{q}$ qui permet d'écrire $e_{c}^{(q, h)}$ à l'aide de la fonction $\Gamma_{p}$ de Jackson définie par

$$
\Gamma_{p}(t)=\frac{(1-t)_{p}^{t-1}}{(1-p)^{t-1}}=(1-p)^{1-t} \frac{(p ; p)_{\infty}}{\left(p^{t} ; p\right)_{\infty}} .
$$

En effet, en remarquant que

$$
z=(1-p) \frac{x}{h}+p=(1-p) \frac{q^{u}-1}{q-1}+p=p\left(p^{-u}-1\right)+p=p^{1-u},
$$

on a

$$
e_{c}^{(q, h)}(x)=h^{c} \frac{\Gamma_{p}(1+c-u)}{\Gamma_{p}(1-u)} .
$$

En utilisant le développement en produit infini de la fonction $\Gamma_{p}$ dans le demiplan $\operatorname{Re} z>0$,

$$
\Gamma_{p}(z)=\prod_{n \geq 1} \frac{\left(1-p^{n+1}\right)^{z}}{\left(1-p^{n+z}\right)\left(1-p^{n}\right)^{z-1}},
$$

on obtient le développement

$$
e_{c}^{(q, h)}(x)=h^{c} \prod_{n \geq 1}\left(\frac{1-p^{n+1}}{1-p^{n}}\right)^{c} \frac{1-p^{n-u}}{1-p^{n+c-u}} .
$$

Le terme général de ce produit s'écrit aussi

$$
\left(\frac{q^{n+1}-1}{q^{n}-1}\right)^{c} \frac{q^{n}-1-(q-1) x / h}{q^{n+c}-1-(q-1) x / h}=\left(\frac{[n+1]_{q}}{[n]_{q}}\right)^{c} \frac{[n]_{q}-x / h}{[n+c]_{q}-x / h}
$$

et tend, quand $q \rightarrow 1^{+}$, vers $\left(\frac{n+1}{n}\right)^{c} \frac{n-x / h}{n+c-x / h}$, terme général de l'expression en produit infini de $\Gamma(1+c-x / h) / \Gamma\left(1-\frac{x}{h}\right)$. Les arguments de convergence sont alors ceux de [7] par exemple.

C3. - La convergence unifome sur tout compact du disque unité, lorsque $q$ tend vers 1 par valeurs réelles positives, de $z \mapsto(z ; p)_{\infty} /\left(p^{c} z ; p\right)_{\infty}$ vers $z \mapsto(1-z)^{c}$ est une conséquence classique du théorème $q$-binomial (où on a utilisé la détermination principale du logarithme). De plus, la famille des $z \mapsto(z ; p)_{\infty} /\left(p^{c} z ; p\right)_{\infty}, p<1$ est normale sur $\mathbb{C} \backslash[1,+\infty[$ : cela résulte du lemme 5 . On en déduit que $z \mapsto(z ; p)_{\infty} /\left(p^{c} z ; p\right)_{\infty}$ converge uniformément sur tout compact de $\mathbb{C} \backslash\left[1,+\infty\left[\right.\right.$ vers $z \mapsto(1-z)^{c}$ lorsque $q \rightarrow 1^{+}$et le résultat suit dans le cas où $h /(q-1) \rightarrow \ell \neq 0$. 
On peut calculer la moyenne des caractères obtenus par passage à la limite dans le cas $\mathrm{C} 1$ (en les considérant comme paramétrés par $\alpha=p_{0}^{\xi}$ ). Nous pouvons envisager deux types de moyennes : celle obtenue grâce à une $q$-intégrale et celle calculée à l'aide d'une intégrale classique (mesure de Lebesgue). Rappelons que, si $a$ et $b$ sont deux nombres réels positifs avec $b>a$ et si $f$ est une fonction à valeurs complexes définie sur $[0, b]$, alors la $q$-intégrale de $f$ entre $a$ et $b$ est donnée par

$$
\begin{aligned}
\int_{a}^{b} f(\alpha) d_{p} \alpha & =\int_{0}^{b} f(\alpha) \mathrm{d}_{p} \alpha-\int_{0}^{a} f(\alpha) \mathrm{d}_{p} \alpha \\
& =(1-p) \sum_{s=0}^{+\infty} f\left(p^{s} b\right) p^{s}-(1-p) \sum_{s=0}^{+\infty} f\left(p^{s} a\right) p^{s} .
\end{aligned}
$$

Dans notre cas, un calcul élémentaire donne la valeur explicite suivante pour la $q$-moyenne :

$$
\int_{p_{0}}^{1} \alpha^{c} \frac{\Theta_{q_{0}, \alpha}}{\Theta_{q_{0}, \alpha p_{0}^{-c}}}(x) \mathrm{d}_{p_{0}} \alpha=\left(1-p_{0}\right) \frac{\Theta_{q_{0}}}{\Theta_{q_{0}, p_{0}^{-c}}}(x)=\left(1-p_{0}\right) e_{c}^{\left(q_{0}, 0\right)}(x) .
$$

En ce qui concerne la seconde moyenne,

$$
\phi(x)=\int_{p}^{1} \alpha^{c} \frac{\Theta_{q, \alpha}}{\Theta_{q, \alpha p^{-c}}}(x) \mathrm{d} \alpha=(-x)^{c} \int_{p}^{1} K\left(-t x^{-1}, c\right) \mathrm{d} t,
$$

nous ne savons pas en faire un calcul explicite. Soulignons cependant qu'elle satisfait l'équation différentielle suivante qui la relie au résultat précédent :

$$
\phi^{\prime}(x)=\frac{c+1}{x} \phi(x)-\frac{1-p}{x} e_{c}^{(q, 0)}(x)
$$

et qu'elle n'est pas définie sur $\mathbb{C}^{*}$ ni même sur son revêtement universel, mais seulement sur le revêtement universel de $\mathbb{C}^{*} \backslash q^{c+\mathbb{Z}}$.

4.1.2. Confluence des logarithmes. - En remarquant que $e_{c}^{(q, h)}(x)$ est holomorphe en $c$, on peut échanger les dérivations successives en $c$ et les opérations de passage à la limite. Reprenant alors les divers arguments de la preuve du théorème 4 on établit la proposition suivante.

Proposition 7. - Soit $k$ un entier strictement positif.

C1 : Pour tout $\varepsilon>0$, la famille $\left(\ell_{c, k}^{\left(q_{0}, h\right)}(x)\right)_{0<h \leq \varepsilon}$ est normale sur $\mathbb{C} \backslash q_{0}^{c} \mathbb{R}^{+}$. La suite $\left(\ell_{c, k}^{\left(q_{0}, h_{n}\right)}\right)_{n \geq 0}$ où $h_{n}=\left(1-p_{0}\right) p_{0}^{n}$ tend vers $\ell_{c, k}^{\left(q_{0}, 0\right)}$, uniformément sur tout compact de $\mathbb{C} \backslash q_{0}^{c} \mathbb{R}^{+}$.

$\mathrm{C} 2$ : La famille $\left(\ell_{c, k}^{(q, h)}\right)_{(q, h) \in \mathcal{Q}}$ converge uniformémement sur tout compact de $\mathbb{C} \backslash\left(c+h_{0} \mathbb{N}^{*}\right)$ vers $\ell_{c, k}^{\left(1, h_{0}\right)}$. 
C3 : Si $\ell \neq 0$, la famille $\left(\ell_{c, k}^{(q, h)}\right)_{(q, h) \in \mathcal{Q}}$ converge uniformément sur tout compact de $\mathbb{C} \backslash \mathbb{R}^{+}$vers $\ell_{c, k}^{(1,0)}$.

4.2. Confluence de la transformation de jauge. - Pour établir le théorème 2, il reste à étudier la partie transformation de jauge. On remarque d'abord qu'en passant à la limite dans les relations

$$
\left\|A_{s}^{(q, h)}\right\| \leq C q^{s-1}\left(\lambda^{-{ }^{[s-1]}} q\right)^{-1}
$$

données par les hypothèses du théorème 2 et en utilisant la continuité en $(q, h) \in \mathcal{Q}^{+}$du majorant, on obtient la première affirmation du théorème grâce à la proposition 1 . L'hypothèse que le système aux $\left(q_{0}, h_{0}\right)$-différences de matrice $A(x)$ est fuchsien signifie que la matrice $C_{0}=I-\left(q_{0}-1\right) A_{0}$ appartient à $\mathrm{Gl}_{n}(\mathbb{C})$. Si ce système est non résonnant, on peut appliquer le théorème 1 à la famille $\Lambda^{\prime}=\Lambda \cup\left\{\left(q_{0}, h_{0}\right)\right\}$ en posant $A^{\left(q_{0}, h_{0}\right)}(x)=A(x)$. Il existe donc une transformation de jauge $F^{\left(q_{0}, h_{0}\right)}(x)$ telle que $A^{F^{\left(q_{0}, h_{0}\right)}}=A_{0}$. La preuve du théorème 2 est alors conséquence du résultat suivant qui établit la continuité de la transformation de jauge.

THÉORÈme 5. - Sous les hypothèses du théorème 2, l'unique transformation de jauge $F^{(q, h)}(x),(q, h) \in \Lambda$ donnée par le théorème 1 converge vers l'unique transformation de jauge $F^{\left(q_{0}, h_{0}\right)}(x)$ tangente à l'identité qui conjugue le système aux $\left(q_{0}, h_{0}\right)$-différences de matrice $A(x)$ à la matrice constante $A_{0}$. La convergence est uniforme sur tout compact de $V^{\left(q_{0}, h_{0}\right)}\left(\lambda^{\prime}\right)$ dans les cas $\mathrm{C} 1$ et $\mathrm{C} 2$, de $V_{\ell}\left(\lambda^{\prime}\right)$ dans le cas $\mathrm{C} 3$.

Démonstration. - Le théorème 1 donne des constantes $C^{\prime}, \lambda^{\prime}>0$ telles que, si $F^{(q, h)}(x)=I+\sum_{s \geq 1} F_{s}^{(q, h)} x^{-{ }^{[s]}} q$, on ait, pour tout $(q, h) \in \Lambda$,

$$
\left\|F_{s}^{(q, h)}\right\| \leq C^{\prime} q^{s-1}\left(\lambda^{\prime-}{ }_{h}^{[s-1]} q\right)^{-1} \leq \frac{C^{\prime}}{\lambda^{\prime}}\left(\lambda^{\prime-}{ }^{[s]} q\right)^{-1}
$$

et donc

$$
\left\|F_{s}^{(q, h)} x^{-{ }^{[}{ }^{[s]} q}\right\| \leq \frac{C^{\prime}}{\lambda^{\prime}} \prod_{k=0}^{s-1} \frac{q^{k} \lambda^{\prime}+h[k]_{q}}{\left|q^{k} x+h[k]_{q}\right|} .
$$

Cette majoration permet de traiter chaque cas comme suit :

C2. - Le changement de variable $x \leftarrow\left(h / h_{0}\right) x$ permet d'appliquer le théorème 3.1 de [3].

C1. - Soient $q_{1} \geq q_{0}$ un majorant de $\{q \mid(q, \lambda) \in \Lambda\}$ et $R>\lambda^{\prime}$. Pour tout $x$ tel que $|x| \geq R$ et tout entier $k \geq 0$, l'inégalité triangulaire renversée jointe à l'inégalité $\left|-{ }_{h}[k]_{q} q^{-k}\right| \leq h /(q-1)$, implique que $\left|x+h q^{-k}[k]_{q}\right| \geq$ 
$R-h /\left(q_{1}-1\right)$. Supposons que $\varepsilon>0$ est assez petit pour que $R-h /\left(q_{1}-1\right)>0$ si $h<\varepsilon$. Alors, en posant $\kappa=1 /\left(q_{1}-1\right)$, on a

$$
\frac{q^{k} \lambda^{\prime}+h[k]_{q}}{\left|q^{k} x+h[k]_{q}\right|}=\frac{\lambda^{\prime}+h q^{-k}[k]_{q}}{\left|x+h q^{-k}[k]_{q}\right|} \leq \frac{\lambda^{\prime}+h \kappa}{R-h \kappa} \leq \frac{\lambda^{\prime}+\varepsilon \kappa}{R-\varepsilon \kappa}=\rho<1
$$

si $\varepsilon$ est assez petit. Ainsi, on peut majorer le terme général de la série $F^{(q, h)}(x)$ par $M \rho^{s}$ et le résultat s'en déduit par convergence dominée.

C3. - Le cas $\ell \in \mathbb{R}^{+}$se traite comme le cas $\mathrm{C} 1$ en remplaçant $\lambda^{\prime}$ par $\lambda^{\prime}-\ell$ (supposé positif). Le cas $\ell=+\infty$ se traite quant à lui comme le cas C2. En effet, on a l'estimation

$$
\left|\frac{q^{k} \lambda+{ }_{h}[k]_{q}}{q^{k} x+{ }_{h}[k]_{q}}\right| \leq \frac{q^{k} \lambda+[k]_{q}}{q^{k} \operatorname{Re} x+[k]_{q}}
$$

et on remarque que le majorant obtenu est indépendant de $h:$ on termine maintenant la preuve par les mêmes raisonnements que pour le cas C2.

Signalons les points suivants. Le cas traité dans [3] est le cas C2 avec $h=1$. Le cas traité dans [8] est le cas C3 avec $q=1$ et donc $\ell=+\infty$. Le cas traité dans [10] est le cas C3 avec $h=0$ et donc $\ell=0$.

Notons pour terminer que le théorème 2 s'applique en particulier lorsqu'on déforme le système non résonnant de matrice

$$
A(x)=\sum_{s \geq 0} A_{s} x^{-h_{0}[s]} q_{0}
$$

où la série converge dans $V^{\left(q_{0}, h_{0}\right)}(\lambda)$ en la famille de systèmes de matrice

$$
A^{(q, h)}(x)=A_{0}^{(q, h)}+\sum_{s \geq 1} A_{s} x^{-h^{[s]} q}
$$

où, si $A_{0}=P \operatorname{diag}\left(A_{c_{1}, r_{1}}^{\left(q_{0}, h_{0}\right)}, \ldots, A_{c_{m}, r_{m}}^{\left(q_{0}, h_{0}\right)}\right) P^{-1}$, alors

$$
A_{0}^{(q, h)}=P \operatorname{diag}\left(A_{c_{1}, r_{1}}^{(q, h)}, \ldots, A_{c_{m}, r_{m}}^{(q, h)}\right) P^{-1} .
$$

\section{BIBLIOGRAPHIE}

[1] A. De Sole \& V. G. KAC - «On integral representations of $q$-gamma and $q$-beta functions », Atti Accad. Naz. Lincei Cl. Sci. Fis. Mat. Natur. Rend. Lincei (9) Mat. Appl. 16 (2005), p. 11-29, arXiv:math.QA/0302032.

[2] A. Duval - «Séries de $q$-factorielles, opérateurs aux $q$-différences et confluence», Ann. Fac. Sci. Toulouse Math. (6) 12 (2003), p. 335-374.

[3] , «Confluence $q$-différence vers différence pour un système fuchsien », Pacific J. Math. 217 (2004), p. 221-245. 
[4] _ , «Une remarque sur les "logarithmes" associés à certains caractères », Aequationes Math. 68 (2004), p. 88-97.

[5] W. A. Harris, JR. - «Linear systems of difference equations », Contrib. Differential Eq. 1 (1963), p. 489-518.

[6] _ _ "Analytic theory of difference equations », in Analytic theory of differential equations (Proc. Conf., Western Michigan Univ., Kalamazoo, Mich., 1970), Lecture Notes in Math., vol. 183, Springer, 1971, p. 46-58. Lecture Notes in Math., Vol. 183.

[7] T. H. KooRnWINDER - « Jacobi functions as limit cases of $q$-ultraspherical polynomials », J. Math. Anal. Appl. 148 (1990), p. 44-54.

[8] J. Roques - «Classification rationnelle et confluence des systèmes aux différences singuliers réguliers », Ann. Inst. Fourier (Grenoble) 56 (2006), p. $1663-1699$.

[9] _ , « Sur les systèmes aux ( $q$-)différences », Thèse, Université Paul Sabatier (Toulouse 3), 2007.

[10] J. SAuloY - « Systèmes aux $q$-différences singuliers réguliers : classification, matrice de connexion et monodromie », Ann. Inst. Fourier (Grenoble) 50 (2000), p. 1021-1071. 